Article

\title{
Fabrication of Flexible, Lightweight, Magnetic Mushroom Gills and Coral-Like MXene-Carbon Nanotube Nanocomposites for EMI Shielding Application
}

\author{
Kanthasamy Raagulan ${ }^{1}{ }^{\mathbb{D}}$, Ramanaskanda Braveenth ${ }^{1}{ }^{\mathbb{D}}$, Lee Ro Lee ${ }^{1}$, Joonsik Lee ${ }^{2}$, \\ Bo Mi Kim ${ }^{3}$, Jai Jung Moon ${ }^{4}$, Sang Bok Lee ${ }^{2, *}$ and Kyu Yun Chai ${ }^{1, *(1)}$ \\ 1 Division of Bio-Nanochemistry, College of Natural Sciences, Wonkwang University, \\ Iksan City 570-749, Korea; raagulan@live.com (K.R.); braveenth.czbt@gmail.com (R.B.); \\ fjrtufl225@naver.com (L.R.L.) \\ 2 Composite Research Division, Korea Institute of Materials Science, Changwon 51508, Korea; \\ astro1228@kims.re.kr \\ 3 Department of Chemical Engineering, Wonkwang University, Iksan 570-749, Korea; 123456@wku.ac.kr \\ 4 Clean \& Science Co., Ltd., Jeongeup 3 Industrial Complex 15BL, 67, 3sandan 3-gil, Buk-myeon 56136, \\ Jeongeup-si 580-810, Korea; jjmoon@cands.kr \\ * Correspondence: leesb@kims.re.kr (S.B.L.); geuyoon@wonkwang.ac.kr (K.Y.C.); \\ Tel.: +82-55-280-3318 (S.B.L.); +82-63-850-6230 (K.Y.C.); \\ Fax: +82-55-280-3498 (S.B.L.); +82-63-841-4893 (K.Y.C.)
}

Received: 20 February 2019; Accepted: 26 March 2019; Published: 2 April 2019

\begin{abstract}
MXenes, carbon nanotubes, and nanoparticles are attractive candidates for electromagnetic interference (EMI) shielding. The composites were prepared through a filtration technique and spray coating process. The functionalization of non-woven carbon fabric is an attractive strategy. The prepared composite was characterized using X-ray photoelectron spectroscopy (XPS), X-ray diffraction (XRD), scanning electron microscope (SEM), energy-dispersive $X$-ray spectroscopy (EDX), and Raman spectroscopy. The MXene-oxidized carbon nanotube-sodium dodecyl sulfate composite (MXCS) exhibited $50.5 \mathrm{~dB}(99.999 \%)$, and the whole nanoparticle-based composite blocked $99.99 \%$ of the electromagnetic radiation. The functionalization increased the shielding by $15.4 \%$. The composite possessed good thermal stability, and the maximum electric conductivity achieved was $12.5 \mathrm{~S} \cdot \mathrm{cm}^{-1}$. Thus, the composite shows excellent potential applications towards the areas such as aeronautics, mobile phones, radars, and military.
\end{abstract}

Keywords: MXene; oxidized carbon nanotube (CNTO); nanoparticle decoration; functionalization; electromagnetic interference (EMI) shielding

\section{Introduction}

Electromagnetic interference (EMI) leads to inevitable interactions in electronic devices. Smaller and high-speed electronic systems are susceptible to issues related to EMI which can affect either adjacent electronic items or humans, thus potentially affecting the security of the nation [1,2]. In war, electromagnetic pulse weapons are being used to affect systems utilizing electromagnetic radiation (EMR) such as radar systems, high tech complex electronic devices, remote control armor, aircraft, and missiles. In addition, EMI affects the functions of sensors in modern electronic vehicles as they transmit signals using weak radiation and microcomputers. Hence, protecting electronic devices from malfunction and achieving electromagnetic compatibility is an essential requirement around the globe. Further, electromagnetic compatibility should be attained by diminishing incoming and outgoing 
electromagnetic radiation, ideally without affecting the function of the devices. This is because EMI not only affects electronic systems but also causes health issues in human beings [3-5].

Various substances have been used for EMI shielding, such as MXenes, graphene (GN), graphene oxide, carbon nanotubes (CNTs), nanoparticles, polymers, fabrics, textiles, composites, and metals in various frequency ranges [2,4-6]. The EMI shielding materials can be categorized in two types: Reflection and absorption domain materials. The reflection domain materials possess mobile charges while absorption domain materials contain magnetic and dielectric materials. The layered and implanted type structures influence the EMI shielding. EMI shielding can be accomplished by suppressing the incident wave, which has the three key mechanisms of absorption, reflection, and multiple reflection. The electric conductivity associated with primary shielding factor is reflection, where the mobile electron interacts with incident wave. The thickness, electric and magnetic dipole loss, magnetic permeability, defects, and structural features induce absorption. The ohmic loss can be achieved by conduction, electron hopping, and tunneling. The polarization loss occurs due to the rearrangement of the polarization while electromagnetic radiation (EMR) is passing through the shielding materials. Polarization can be induced by embedding functionalities, hybrid fillers, nanofillers, and defects in the matrix of the composite. The inhomogeneous scattering centers, layered structure, hollow structure, and interfaces generate multiple reflection, which finally leads to absorption. Further, the skin depth limits the EMI shielding effectiveness, which should be lower than that of the thickness of EMI shielding materials [2,6-11]. Certain properties, such as being lightweight, conductive, corrosion resistant, flexible, cost-effective, and high strength, are preferable for a modern EMI shielding material. Metals are conventionally exploited as shielding materials due to the fact that they possess excellent conductivity, but they are unable to fulfill the current needs of compact electronic systems. Hence, carbon-based substances are attracting attention, as their properties can be tuned by incorporating other materials like nanoparticles and polymers [1-5,8]. In addition, incorporating carbon nanotubes (CNTs), MXenes, polymers, and graphene with non-woven carbon fabric significantly increases EMI shielding. Thus, establishing a conductive network is an essential factor for good EMI shielding [5,8,12]. Different combinations of the constituents and amounts of filler loading have been used to fabricate multi-functional EMI shielding materials [13-17].

MXenes $\left(M_{(n+1)} X_{n} T_{x}\right)$ are two dimensional (2D) material derived from a corresponding three dimensional (3D) MAX phase $\left(\mathrm{M}_{(\mathrm{n}+1)} \mathrm{AX}\right.$ ), where $\mathrm{M}$ is early transition elements $(\mathrm{Ti}, \mathrm{V}, \mathrm{Cr}, \mathrm{Nb}, \mathrm{Ta}$, $\mathrm{Zr}$, and Mo), A includes group 13/14 elements, $\mathrm{X}$ represents carbon or nitrogen, and $\mathrm{T}_{\mathrm{x}}$ is surface functional groups $(-\mathrm{OH},-\mathrm{F}$, and $=\mathrm{O})$ [2]. A selective etching strategy is used in the production of MXenes. The minimally intensive layer delamination (MILD) method $(\mathrm{LiF} / \mathrm{HCl})$ has recently been endorsed, as it abridges the synthetic process, and $\mathrm{HF}, \mathrm{NH}_{4} \mathrm{HF}_{2}$, and $\mathrm{FeF}_{3} / \mathrm{HCl}$ have also been practiced. During the etching, the weaker $\mathrm{M}-\mathrm{A}$ bonds are eradicated while the strong $\mathrm{M}-\mathrm{C}$ bond remains with newly formed functionalities $[2,18]$. MXenes have a metal-like nature, and similar to graphene, have been used for various purposes such as in sensors, capacitors, storage, and EMI shielding materials. In general, MXenes are hydrophilic in nature, as $-\mathrm{OH}$ is one of the surface functional groups. Thus, MXenes can be incorporated with various materials like polymers in order to tune their properties [2]. There are various types of MXenes that have been studied such as $\mathrm{Nb}_{2} \mathrm{CT}_{\mathrm{x}}, \mathrm{Ti}_{3} \mathrm{CNT}_{\mathrm{x}}$, and $\mathrm{Ti}_{2} \mathrm{CT}_{\mathrm{x}}$. In addition, MXenes are used to make hybrid composites such as $\mathrm{TiO}_{2}-\mathrm{Ti}_{3} \mathrm{C}_{2} \mathrm{~T}_{\mathrm{X}} /$ graphene, $\mathrm{Ti}_{3} \mathrm{C}_{2} \mathrm{~T}_{\mathrm{X}}$-sodium alginate, $\mathrm{Ti}_{3} \mathrm{C}_{2} \mathrm{Tx} / \mathrm{PVA}$, cellulose nanofibers- $\mathrm{Ti}_{3} \mathrm{C}_{2} \mathrm{~T}_{\mathrm{x}}$ and $\mathrm{Ti}_{3} \mathrm{C}_{2} \mathrm{~T}_{\mathrm{X}}$ /paraffin. MXenes exhibit maximum EMI shielding of $92 \mathrm{~dB}$ with $45 \mu \mathrm{m}$ thickness. Thus, the loading amount of MXenes in different polymers matrices, the morphology of composite, and the thickness influence the EMI shielding of the MXene [19-23].

In this study, we developed a layered pliable composite with different surface morphology and magnetic composite. Each layer of the composite consists of a layered and magnetic domain for which we employed spray coating and filtration technique under gravity. The functionalization of carbon fabric intercalation of the MXene, CNTs, and magnetic nanoparticle dramatically changed the EMI shielding. The spray coating samples were denoted as MXCNTCx, where $x(x=10,25$, or 30$)$ was 
the number of coating cycles, and Ni coated fabric was expressed as MXCNTNi25. The composite fabricated using the filtration method was denoted as $\mathrm{MXCBCM,} \mathrm{where} \mathrm{M}$ is the type of metal nanoparticle such as $\mathrm{Ni}, \mathrm{Co}, \mathrm{Fe}, \mathrm{Cu}$, and $\mathrm{Fe}_{3} \mathrm{O}_{4}$. Its corresponding composites were $\mathrm{MXCBCNi}$, MXCBCCo, MXCBCFe, MXCBCCu, and MXCBCFeO, respectively. Further, MXCB and MXCS were labelled based on the surfactant used, like cetyltrimethylammonium bromide (CTAB) and sodium dodecyl sulfate (SDS), respectively. MC and FC indicated uncoated carbon fabric and functionalized carbon fabric, respectively. The parameters of EMI shielding, elementals analysis, morphology, structural analysis, electric conductivity, surface property, magnetic property, and thermal stability were investigated in detail.

\section{Materials and Methods}

\subsection{Materials}

Multiwall carbon nanotube (MWCNTs) (CM-90, $90 \mathrm{wt} \%$, diameter of $20 \mathrm{~nm}$, and length of $100 \mu \mathrm{m})$ were purchased from Applied Carbon Technology Co. Ltd. (Pohang, Korea). Carbon fiber (fiber diameter 7-micron, $6 \mathrm{~mm}$ length) and polyethylene terephthalate (PET) binder (fiber diameter $2.2 \mathrm{dtex}, 5 \mathrm{~mm}$ ) were collected from TORAY product, (Tokyo, Japan). Sodium dodecyl sulfate (SDS) (98\%), lithium fluoride (LiF) $\left(98 \%, 300\right.$ mesh), sodium borohydride $\left(\mathrm{NaBH}_{4}\right)$, polyacrylamide (PAM), anhydrous $\mathrm{FeCl}_{2}, \mathrm{FeCl}_{3}, \mathrm{NiCl}_{2}, \mathrm{CuCl}_{2}, \mathrm{CoCl}_{2}$, and cetyltrimethylammonium bromide (CTAB) were obtained from Sigma Aldrich (Seoul, Korea). Wet laid nickel coated non-woven carbon fabric (basic density of $19.2 \mathrm{~g} / \mathrm{m}^{2}$, thickness of $150 \mu \mathrm{m}$ ) was acquired from Clean \& Science Co. Ltd., (Seoul, Korea). Nitric acid $\left(\mathrm{HNO}_{3}-70 \%\right)$ and hydrochloric acid ( $\left.\mathrm{HCl}-35 \%\right)$ were obtained from Samchun Chemical Co., Ltd. (Seoul, Korea). Chitooligosaccharide (Mw 5000) was issued by biomedical polymer lab, Sunchon National University (Suncheon, Korea).

\subsection{Synthesis of $\mathrm{Ti}_{3} \mathrm{AlC}_{2}$}

We reported that $\mathrm{TiC}, \mathrm{Ti}$, and $\mathrm{Al}$ powders were taken with molar ration of 2:1:1 ball milled by using Pulverisette 6 Planetary Mono Mill (Fritsch, Germany) in ethanol medium at $200 \mathrm{rpm}$ for $1 \mathrm{~h}$ in a nitrogen environment. The resultant mixture was dried at $80^{\circ} \mathrm{C}$ for $12 \mathrm{~h}$. Then, $3 \mathrm{~g}$ of $12 \mathrm{~mm}$ diameter disc was prepared by applying $27.6 \mathrm{MPa}$ pressure for $5 \mathrm{~min}$ in a laboratory press. The resultant disc was treated at $1350{ }^{\circ} \mathrm{C}$ with a heating rate of $20^{\circ} \mathrm{C} /$ minute in argon gas for $2 \mathrm{~h}$, then cooled down to room temperature. The treated disc was again ball milled in ethanol medium at $300 \mathrm{rpm}$ for $3 \mathrm{~h}$ in a nitrogen environment. The powder yield was then dried at $80^{\circ} \mathrm{C}$ for $12 \mathrm{~h}$, and the obtained product was directly used for MXene synthesis [24].

\subsection{Preparation of Oxidized Carbon Nanotube (CNTO)}

$1 \mathrm{~g}$ of MWCNT (CNT) and $90 \mathrm{~mL}$ of $\mathrm{HNO}_{3}$ were sonicated by using a mini ultrasonic cleaner (Uil ultrasonic Co., Ltd., Gyeonggi-do, Republic of Korea) for $5 \mathrm{~h}$ at room temperature. The volume of the reaction mixture was doubled by adding deionized (DI) water and filtered. The acid solution and reacted CNT were separated by filtration. The resultant black color product known as CNTO was washed until reaching a neutral $\mathrm{pH}$, then dried at $80^{\circ} \mathrm{C}$ for $24 \mathrm{~h}$. This $\mathrm{CNTO}$ was used for the coating process and synthesis decorated CNTO.

\subsection{Preparation of $\mathrm{Fe}_{3} \mathrm{O}_{4}$ Decorated $\mathrm{CNTO}$}

$100 \mathrm{~mL}$ of $0.1 \mathrm{M}$ of $\mathrm{Fe}^{3+}, 100 \mathrm{ml}$ of $0.1 \mathrm{M}$ of $\mathrm{Fe}^{2+}$, and $0.5 \mathrm{~g} \cdot \mathrm{L}^{-1}$ of $\mathrm{CNTO}$ were stirred together at $30{ }^{\circ} \mathrm{C}$ for $30 \mathrm{~min}$. A total of $10 \%$ of $\mathrm{NH}_{4} \mathrm{OH}$ was added dropwise until the $\mathrm{pH}$ of the solution reached $11-12$, along with $2 \mathrm{~mL}$ of $0.2 \mathrm{M}$ SDS. Then, the temperature of the reaction mixture was raised up to $80^{\circ} \mathrm{C}$ and stirred for $2 \mathrm{~h}$. The volume of the solution was reduced by half via evaporation and then cooled down to room temperature. The resultant product was washed until reaching a neutral $\mathrm{pH}$ in a vacuum filter, then dried at $105^{\circ} \mathrm{C}$ for $12 \mathrm{~h}$. 


\subsection{Preparation of Nanoparticle Decorated CNTO}

$100 \mathrm{~mL}$ of $0.1 \mathrm{M}^{2+}$ of metal ion solution $\left(\mathrm{Fe}^{2+}, \mathrm{Ni}^{2+}, \mathrm{Cu}^{2+}\right.$, and $\left.\mathrm{Co}^{2+}\right)$ and $0.5 \mathrm{~g} \cdot \mathrm{L}^{-1}$ of CNTO were

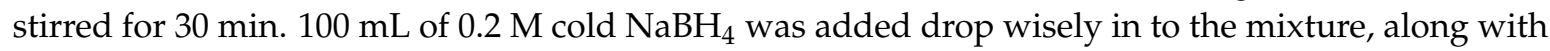
$0.2 \mathrm{~g} \cdot \mathrm{L}^{-1}$ of SDS. Fe, Ni, Co, and $\mathrm{Cu}$ decorated CNTO were successfully synthesized in a nitrogen environment. The product was filtered and washed by an ample amount of DI water. Then, the product was dried at $80{ }^{\circ} \mathrm{C}$ and $0.8 \mathrm{~atm}$ in a vacuum oven for $12 \mathrm{~h}$. The obtained product was denoted as $\mathrm{CM}$, where $\mathrm{C}$ is $\mathrm{CNTO}$ and $\mathrm{M}$ is nanoparticles. The corresponding decorated CNTOs were denoted as $\mathrm{CFe}$, $\mathrm{CNi}, \mathrm{CCO}$, and $\mathrm{CCu}$.

\subsection{Preparation of Dispersed Solutions}

$0.1 \mathrm{~g}$ of CNTO and $0.1 \mathrm{~g}$ of SDS were mixed in $100 \mathrm{~mL}$ of deionized water and sonicated for $3 \mathrm{~h}$. Then, the dispersed mixture was refluxed at $120{ }^{\circ} \mathrm{C}$ for $12 \mathrm{~h}$. The obtained well dispersed solution was then used for the spray coating process. In addition, $0.4 \mathrm{~g}$ of CNTO and $0.4 \mathrm{~g}$ of CTAB were added together in $100 \mathrm{~mL}$ of deionized water and sonicated for $5 \mathrm{~h}$ (CTAB-CNTO). This procedure was repeated using SDS and CNTO (SDS-CNTO). The decorated nanoparticle dispersed solution was prepared accordingly; equal amounts of CTAB and CM $(0.1 \mathrm{~g})$ were sonicated in $25 \mathrm{~mL}$ of deionized water for $30 \mathrm{~min}$. The obtained dispersed solutions were directly used for the filtration process.

\subsection{Preparation of MXene and MXene Colloidal Solution}

Equal amounts $(1 \mathrm{~g})$ of $\mathrm{Ti}_{3} \mathrm{AlC}_{2}$ and $\mathrm{LiF}$ were mixed together in $20 \mathrm{~mL}$ of $9 \mathrm{M} \mathrm{HCl}$ solution. This mixture was stirred at $35{ }^{\circ} \mathrm{C}$ for $24 \mathrm{~h}$. The etched product was washed with DI water up to approximately $\mathrm{pH} 6$ by centrifuging at $3500 \mathrm{rpm}$ for $5 \mathrm{~min}$, then $\mathrm{Ti}_{3} \mathrm{C}_{2} \mathrm{~T}_{\mathrm{x}}$ was dried in a vacuum oven for $12 \mathrm{~h}$. Then, $0.1 \mathrm{~g}$ of MXene was sonicated in $10 \mathrm{~mL}$ of DI water in a nitrogen environment at about $15-17{ }^{\circ} \mathrm{C}$ for $2 \mathrm{~h}$. The sonicated MXene solution was centrifuged at $3500 \mathrm{rpm}$ for $30 \mathrm{~min}$, and the supernatant was collected in a Teflon container stored at $5{ }^{\circ} \mathrm{C}$ for the coating process. The concentration of the MXene colloidal solution was $0.175 \mathrm{~g} \cdot \mathrm{L}^{-1} .100 \mathrm{~mL}$ of colloidal solution was filtered by using $0.45 \mu \mathrm{m}$ of $47 \mathrm{~mm}$ of Nylon supported filter paper and dried at $80^{\circ} \mathrm{C}(0.8 \mathrm{~atm})$ until obtained constant weight. The concentration was calculated based on the weight differences.

\subsection{Synthesis of Carbon Fabric by Wet Laid Method (MC)}

We reported that $0.6 \mathrm{~kg}$ of carbon fiber, $0.15 \mathrm{~kg}$ of PET binder and 0.3 weight percent of dispersant (PAM) were dispersed in a sufficient amount of deionized (DI) water at $500 \mathrm{rpm}$ for $10 \mathrm{~min}$. The general wet laid method was used to produce the web. During the process, the drum dryer was used with a $140{ }^{\circ} \mathrm{C}$ surface temperature and $7 \mathrm{~m} \cdot \mathrm{min}^{-}$speed. The areal density of obtained fabric was $30 \mathrm{~g} \cdot \mathrm{m}^{-2}[24]$.

\subsection{Fabrication of Fabric Composite}

\subsubsection{Preparation of Functionalized Carbon Fabric (FC)}

$0.2 \mathrm{~g}$ of chitooligosaccharide was dissolved in $100 \mathrm{~mL}$ of deionized water and stirred for $15 \mathrm{~min}$. Then, carbon fabric with a dimension of $29 \times 21 \mathrm{~cm}^{2}$ and a basic density of $30 \mathrm{~g} \cdot \mathrm{m}^{-2}$ was dipped in the chitooligosaccharide solution for $2 \mathrm{~min}$ and dried at $100{ }^{\circ} \mathrm{C}$ for $12 \mathrm{~h}$. This fabric was directly used for the preparation of the filtration-based composite.

\subsubsection{Fabrication of MXCNTCx Composite by Spray Coating}

A series of MXene-CNTO composites were prepared by the spray coating process, for which $30 \mathrm{~g} \cdot \mathrm{m}^{-2}$ of MC with a dimension of $29 \times 21 \mathrm{~cm}^{2}$ was used, where one CNTO layer was sandwiched between two MXene layers. The thickness of the fabric was adjusted by a spraying and drying process. The drying was done using an air-drying gun while spraying was done by using an air compressor (Keyang compressors (KAC-25), Sichuan, China). This process was repeated for Ni coated 
fabric in order to compare the EMI shielding of carbon fabric. The coating on carbon fabric was denoted as MXCNTCx, while the Ni coated carbon fabric was denoted as MXCNTNiCx, where $x$ was the number of coating cycles. MXCNTC30, MXCNTC25, MXCNTC10 and MXCNTNiC25 were successfully manufactured.

\subsubsection{Fabrication of MXene-CNTO Composite by Filtration}

$100 \mathrm{~mL}$ of MXene colloidal solution, $100 \mathrm{~mL}$ of CM dispersed solution and $70 \mathrm{~mL}$ of dispersed CNTO were alternatively filtered through FC under gravity and dried using an air gun. The resultant composite was denoted as MXCBCM. Further, MXCB was prepared by using $100 \mathrm{~mL}$ of MXene colloidal solution and $70 \mathrm{~mL}$ of CTAB-CNTO dispersed while $100 \mathrm{~mL}$ of MXene colloidal solution and $70 \mathrm{~mL}$ of SDS-CNTO dispersed mixture were used to prepare MXCS. Finally, MXCBCFeO, MXCBCFe, MXCBCNi, MXCBCCo, and MXCBCCu were successfully prepared.

\subsection{Characterization}

The structural features of the composites were investigated using a high-resolution Raman spectrophotometer (Jobin Yvon, LabRam HR Evolution (Horiba, Tokyo, Japan). A Laser Flash Apparatus LFA457 (NETZSCH, Wittelsbacherstrabe, Germany) was used to measure the density of the composites. A field emission scanning electron microscope (SEM, S-4800 (Hitachi, Tokyo, Japan) was used to examine the surface morphology of the composites. XPS with a 30-400 $\mu \mathrm{m}$ spot size at $100 \mathrm{~W}$ of Emax (Al anode) (K-Alpha, Thermo Fisher, East Grinstead, UK) was used to analyze the chemical environment and elemental percentage of the composites. A High-power X-ray Diffractometer $\mathrm{D} / \mathrm{max}-2500 \mathrm{~V} / \mathrm{PC}$, (Ragaku, Tokyo, Japan) with $\mathrm{Cu}(\mathrm{K} \alpha)$ was used to record the X-ray diffraction patterns of the composites. The EMI shielding effectiveness (SE) of the composite in S-band (1-3 GHz) was recorded using an EMI shielding tent ASTM-D4935-10, ASTM International (West Kentucky, PA, USA) at room temperature while X-band (8.2-12.4 GHz) EMI shielding was measured using a vector network analyzer (VNA, Agilent N5230A, Agilent Technologies, Santa Clara, CA, USA) with a sample size of $22.16 \mathrm{~mm} \times 10.16 \mathrm{~mm}$. The four-probe method FPP-RS8, DASOL ENG (Seoul, Korea) was used to measure the electric conductivity of the composites. The thermal stability of the composites was tested using a Thermal Analyzer DSC TMA Q400 (TA Instruments Ltd., New Castle, DE, USA). A Mitutoyo thickness 2046S dial gage (Mitutoyo, Kanagawa, Japan) was used to measure the thickness of the composites. The surface property was measured using a Phoenix-300A contact angle meter (S.E.O.Co., Ltd., Suwon, Korea). The magnetic property was measured using SQUID_VSM (Quantum Design, Inc., San Diego, CA, USA). The graphs were plotted using a Savitzky-Golay function (Origin 2017 graphing and analysis, Origin Lab (Boston, MA, USA).

\section{Results and Discussion}

\subsection{Structural Analysis}

\subsubsection{Scanning Electron Microscopic Analysis of Morphology}

SEM was used to characterize the morphology of nanoparticles and composites, the arrangement of CNTO, nanoparticles, and MXene flakes, the structural feature of fiber, and the topography of the composites. Figure 1 illustrates the differently decorated CNTOs by different types of nanoparticles. The oxidation of the carbon nanotube mainly occurred in the tip of the carbon nanotube, as confirmed by the SEM images (Figure 1 and Figure S1), and the decoration of carbon nanotube generated a cauliflower-like structure (Figure $1 \mathrm{a}-\mathrm{c}, \mathrm{e}$ and Figure S1a,b,d). The oxidized carbon nanotube consists of a carboxylic acid functional group which can act as anchoring side of the nanoparticles. According to the Figure 1 and Figure S1, the deposition of the nanoparticle grafting occurred in the terminal of the $\mathrm{CNTO}$, indicating that the oxidation predominantly happed in the tip. The oxidation of multiwall carbon nanotube produced the terminal carboxylic group which helps terminal grafting, inhibits the 
aggregation and increases the solubility in water. This phenomenon leads the various morphologies in decorated CNTOs $[25,26] . \mathrm{Fe}_{3} \mathrm{O}_{4}, \mathrm{Fe}$, and $\mathrm{Cu}$ nanoparticles behaved in a similar manner, whereas the Ni nanoparticle encircled all of the carbon nanotube and was densely packed like a cauliflower. The self-assembling of the Co nanoparticles was completely different from that of another nanoparticle used. This is because it consumed CNTO as a template and formed a structure-like bacterial chain (Figure $1 \mathrm{~d}$ and Figure S1c) [27]. The precursor of $\mathrm{MXene}$, which is $\mathrm{Ti}_{3} \mathrm{AlC}_{2}$, exhibited a layered structure (Figure S3h) [6]. The MILD etching created cleaves, due to the eradication of the Al layer and the evolution of the hydrogen gas (Figure $1 \mathrm{f}$ and Figure S3i,j). This gap is more prominent in the clay etching method (50\% of HF) [18]. The etched MXene showed a layered structure with fewer gaps, and the folding of the single flake confirmed that the etching occurred. As it consisted of small gaps, it appeared like a MAX phase (Figure 1f and Figure S3i,j). Further, the presence of a single MXene flake in the composites affirmed the occurrence of effective exfoliation during the process (Figures $1 f$ and 2b-i) [27].

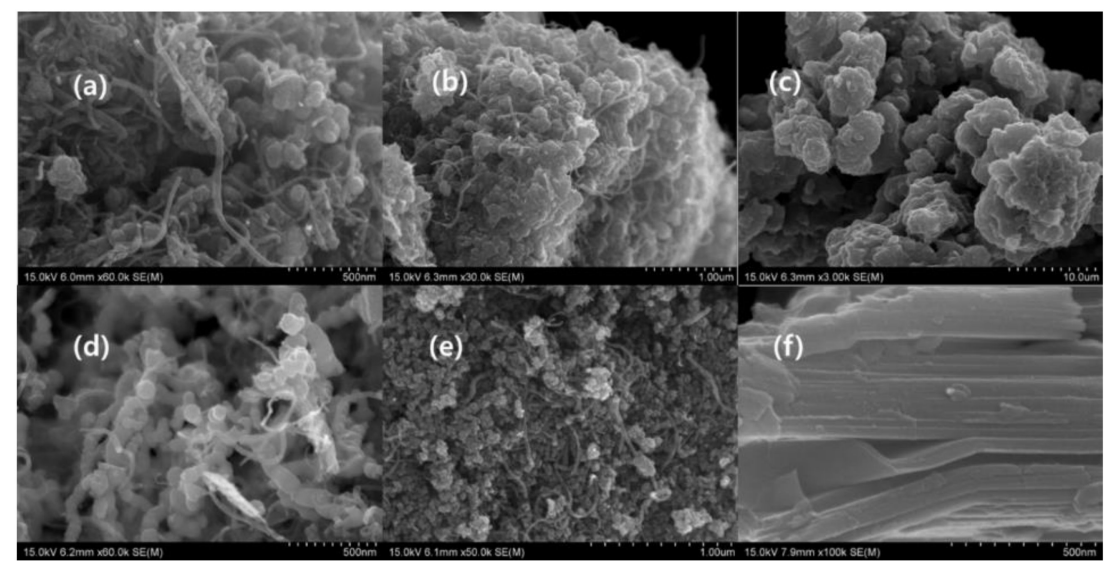

Figure 1. SEM image of oxidized carbon nanotubes (CNTOs) decorated by (a) $\mathrm{Fe}_{3} \mathrm{O}_{4}(\times 60,000)(\mathbf{b}) \mathrm{Fe}$ $(\times 30,000)(\mathbf{c}) \mathrm{Ni}(\times 3000)(\mathbf{d}) \mathrm{Co}(\times 60,000)(\mathbf{e}) \mathrm{Cu}(\times 50,000)$ and $(\mathbf{f}) \mathrm{Ti}_{3} \mathrm{C}_{2} \mathrm{~T}_{\mathrm{x}}(\times 100,000)$.

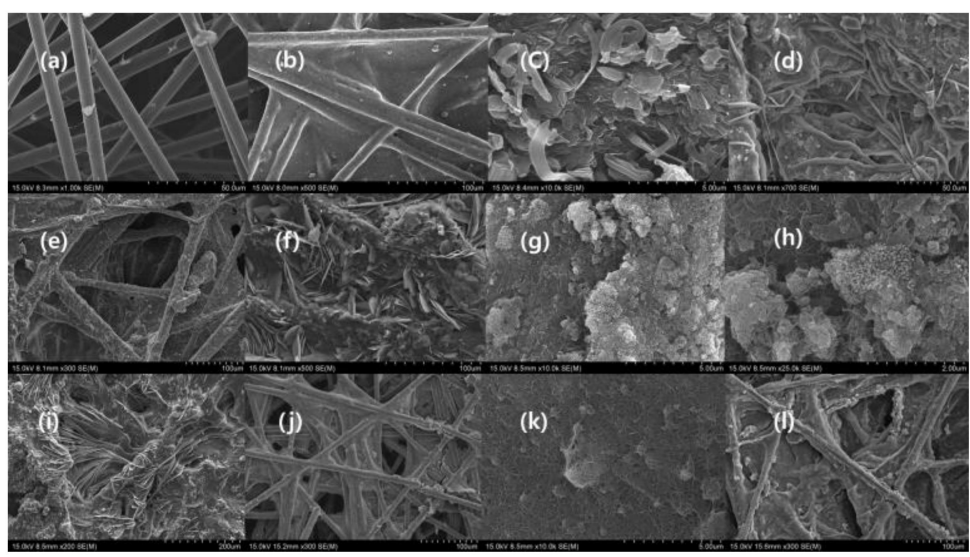

Figure 2. SEM image of carbon fabric composite of (a) Functionalized carbon fabric $(\mathrm{FC})(\times 1000)$ (b) $\mathrm{MXCB}(\times 500)(\mathbf{c})$ surface of MXCS $(\times 10,000)(\mathbf{d}) \mathrm{MXCBFeO}(\times 700)(\mathbf{e}) \mathrm{MXCBFe}(\times 500)(\mathbf{f}) \mathrm{MXCBNi}$ $(\times 500)($ g) MXCBCCo $(\times 10,000)($ h) MXCBCCo $(\times 25,000)(\mathbf{i}) \mathrm{MXCBCCu}(\times 200)(\mathbf{j}) \mathrm{MXCNTC25}(\times 300)$ (k) MXCNTC25 $(\times 10,000)$ and $(\mathbf{l})$ MXCNTNi25 $(\times 300)$.

Figure 2 illustrates the morphology of the composites. The functionalized nonwoven carbon fabric exhibited a similar morphology of nonwoven carbon fabric, where the fibers were arranged capriciously (Figure 2b,j and Figure S3d). The fibers of the carbon fabric possessed annular gaps and cracks which were occupied by CNTO, MXene, and nanoparticle [5]. This functionalization and the nanomaterials altered the property of $\mathrm{MC}$ and dramatically changed the structural feature of the carbon fabric (Figure 2a-1). Filtration was an effective strategy over spray coating, because 
filtration closed most of the gaps between fibers and interconnected the fibers with fewer defects, while MXCNTNiC25 had prominent defects (Figure 21 and Figure S3b-j) [6]. MXCB formed like a film with well interconnected fibers, whereas few pore structures remained (Figure $2 \mathrm{~b}$ and Figure $\mathrm{S} 2 \mathrm{~b}$ ). MXene, $\mathrm{CNTO}$, and $\mathrm{Fe}_{3} \mathrm{O}_{4}$ decorated CNTOs formed a structure like roots of a tree fixed on the soil surface, and some points of the MXene flake formed an unexfoliated MXene structure. Further, a root-like nature was given by the MXene flakes (Figure 2d). MXCBFe and MXCNTC25 formed similar structures and MXCBFe generated a highly interconnected network. In addition, the MXene flakes self-assembled in a random manner and showed a similar pattern of graphene-Polyvinylidene fluoride (PVDF) coated fabric (Figure 2c,f) [6]. Furthermore, MXCBCCu exhibited a mushroom gills-like structure which was generated by MXene flakes arranged in parallel among nanoparticles and CNTO [28]. The MXCBCCo and CNi exhibited a coral like morphology (Figure 2g,h) [29]. The coral structure was formed by cubic Co nanoparticles. In order to achieve the coral structure, CCo was used as mediator (Figure S2h,i). The surface of the MXCNTC25 displayed a network of CNTO encircling the MXene flake. Furthermore, the fibers in MXCNTNiC25 were interconnected with many defects and cracks which dramatically affected the EMI shielding and conductivity (Figure 2k,l). The etching caused the introduction of new elements such as $\mathrm{F}, \mathrm{Cl}$, and $\mathrm{O}$ while it eradicated most of the $\mathrm{Al}$ from the MAX phase, leaving Ti and $\mathrm{C}$ (Table S1). In addition, $\mathrm{Cl}, \mathrm{F}$, and $\mathrm{O}$ were derived from etchant. According to the EDX analysis, the $\mathrm{O}$ and $\mathrm{C}$ were major elements present in all composites, while other metals like $\mathrm{Co}, \mathrm{Ni}, \mathrm{Fe}$, and $\mathrm{Cu}$ were also present based on the precursor used to manufacture composites. As the MXene was a structural unit of the composites, the Ti and F prevailed in most of the composites, and $\mathrm{Al}$ and $\mathrm{Br}$ were also found in some composites, which are derived from MXene colloidal solution and CTAB surfactant, respectively. In addition, exfoliated MXene had more than a single layer, which consisted of the little amount of remaining Al. Further, spray coated fabric consisted of S, which was derived from the SDS surfactant used (Table S1 and Figure S2c).

\subsubsection{Raman Spectroscopic Analysis for Structure of Carbon-Based Material}

The structural and crystalline nature of materials like MXene, graphene, and CNT can be investigated using Raman spectroscopy [2,5]. Figure 3a,b illustrate the Raman spectrum of the decorated carbon nanotube and composites as plotted between 250 and $3500 \mathrm{~cm}^{-1}$ Raman shifts. The peaks at 624,394 , and $263 \mathrm{~cm}^{-1}$ were attributed to the in-plane vibrational mode of surface functionalities, $\mathrm{C}$, and $\mathrm{Ti}$, respectively. In addition, $\mathrm{Ti}_{3} \mathrm{C}_{2} \mathrm{~T}_{\mathrm{x}}$ engendered feeble wide $\mathrm{D}$ and $\mathrm{G}$ bands at 1353 and $1568 \mathrm{~cm}^{-1}$, respectively, and the peaks at 624,510 , and $398 \mathrm{~cm}^{-1}$ exhibited the presence of $\mathrm{TiO}_{2}$ anatase (Figure S4k) [30-33]. In addition, the missing peak at $263 \mathrm{~cm}^{-1}$ revealed the absence of the $\mathrm{Al}$ layer and the fixing of new surface functionalities in the eradicated Ti-C-Al bond. The $\mathrm{G}$ band and $\mathrm{G}^{\prime}$ band of the CNT and CNTO were located at 1570.9 and $2675.9 \mathrm{~cm}^{-1}$, respectively. The position of the D band slightly differed from that of the CNT peak located at $1336.3 \mathrm{~cm}^{-1}$ while CNTO generated a peak at $1341.5 \mathrm{~cm}^{-1}$. In addition, CNTO gave rise to new weak peaks at 2435.5, 2916.2, and $3320.4 \mathrm{~cm}^{-1}$ while CNT formed weak peaks at 2420, 2371, and $3226.2 \mathrm{~cm}^{-1}$. These differences were created due to the oxidation that was considered as oxidational effect of the carbon nanotube. Furthermore, the presence of defects and the amorphous nature of CNT generated the D band while the graphite structure induced the $G$ band. The characteristic $G^{\prime}$ band at $2672 \mathrm{~cm}^{-1}$ was formed by an overtone of the $\mathrm{D}$ band. Further, the level of defect present in the carbon nanotube can be explained using the ratio between $\mathrm{I}_{\mathrm{D}} / \mathrm{I}_{\mathrm{G}}$ and $\mathrm{I}_{\mathrm{D}} / \mathrm{I}_{\mathrm{G}^{\prime}}[34-45] . \mathrm{I}_{\mathrm{D}} / \mathrm{I}_{\mathrm{G}}$ and $\mathrm{I}_{\mathrm{D}} / \mathrm{I}_{\mathrm{G}^{\prime}}$ of $\mathrm{CNT}$ were 0.79 and 1.39 , respectively, while those of CNTO were 0.96 and 1.76 , respectively. The CNTO possessing higher values of $\mathrm{I}_{\mathrm{D}} / \mathrm{I}_{\mathrm{G}}$ and $\mathrm{I}_{\mathrm{D}} / \mathrm{I}_{\mathrm{G}^{\prime}}$ revealed that $\mathrm{CNTO}$ had more defect density than $\mathrm{CNT}$. Hence, chemical oxidation created disorder in the carbon nanotube. The D band of the CNTO and nanoparticle decorated CNTO was located between $1340-1355 \mathrm{~cm}^{-1}$ while the $G$ and $G^{\prime}$ bands were placed between $1570-1585 \mathrm{~cm}^{-1}$ and $2675-2700 \mathrm{~cm}^{-1}$, respectively. All of the carbon nanotubes and decorated CNTOs exhibited weak peaks between $2910-2943 \mathrm{~cm}^{-1}$ and $3220-3240 \mathrm{~cm}^{-1}$, respectively. Further, there were extra peaks at lower Raman shift, which were due to the carbon-metal and oxygen-metal vibration modes 
(Figure 3a and Table S2) [46,47]. The G band intensity of CNTO was lower than that of CNT when compared with its corresponding $\mathrm{D}$ band. A similar pattern was shown by $\mathrm{CFe}$ whereas the other decorated CNTOs exhibited a higher D band intensity, implying that the introduction of nanoparticle generates the defect. The $\mathrm{I}_{\mathrm{D}} / \mathrm{I}_{\mathrm{G}}$ values of $\mathrm{CCu}, \mathrm{CCo}, \mathrm{CNi}, \mathrm{CFe}, \mathrm{CFeO}$, and FC were 1.01, 1.15, 1.02, $0.92,1.4$, and 0.94 , respectively, whereas the corresponding $\mathrm{I}_{\mathrm{D}} / \mathrm{I}_{\mathrm{G}^{\prime}}$ values were $1.84,1.96,1.68,1.74$, 2.71 , and 4.18, respectively [47]. All of the fabric showed a similar Raman spectra pattern and a peak originated between $1339-1350 \mathrm{~cm}^{-1}$ which was responsible for D band of the composites, whereas the corresponding $G$ and $G^{\prime}$ bands laid between $1567-1586 \mathrm{~cm}^{-1}$ and $2678-2688 \mathrm{~cm}^{-1}$, respectively. The non-woven carbon fabric gave rise to $D$ and $G$ bands at 1363 and $1592.1 \mathrm{~cm}^{-1}$, respectively, which is due to the graphite (HOPG), indicating the presence of the graphite-like structure and the generation of a feeble $\mathrm{G}^{\prime}$ band at $2908.2 \mathrm{~cm}^{-1}$. The $\mathrm{I}_{\mathrm{D}} / \mathrm{I}_{\mathrm{G}}$ values of the MC, MXCS, MXCBCCu, MXCBCCo, MXCBCNi, MXCBCFe, MXCBCFeO, and MXCB were 0.91, 1.03, 0.81, 0.87, 1.01, 0.86, 0.81, and 1.01, respectively, and the corresponding $\mathrm{I}_{\mathrm{D}} / \mathrm{I}_{\mathrm{G}^{\prime}}$ values were 4.13, 2.33, 1.48, 2.12, 1.88, 1.96, 2.09, and 2.04, respectively (Figure $3 b$ and Table $S 2$ ). All of the composite $\mathrm{I}_{\mathrm{D}} / \mathrm{I}_{\mathrm{G}}$ values were relatively similar to $\mathrm{MC}$, while the decreasing $\mathrm{I}_{\mathrm{D}} / \mathrm{I}_{\mathrm{G}^{\prime}}$ value of composite confirmed that the defects of the fabric were diminished significantly. The disappearing of the lower Raman shift of MXene and decorated carbon nanotube and the formation of the new peaks confirmed that the proper link occurred between the fabric, MXene, carbon nanotube, and nanoparticles.

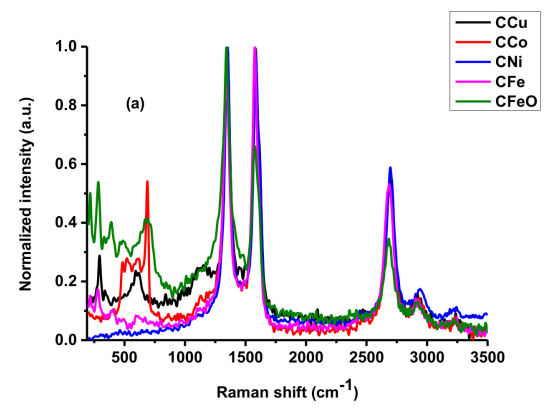

(a)

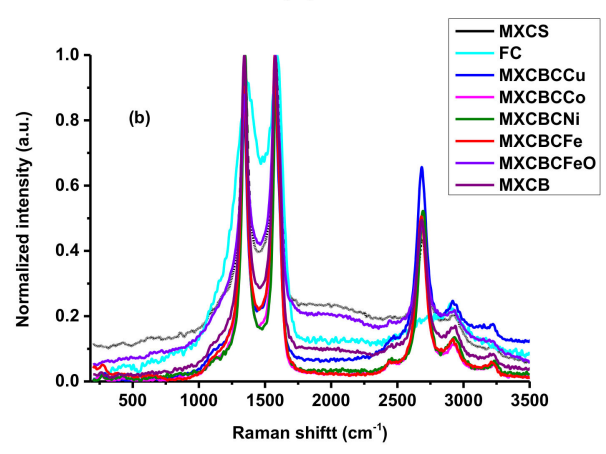

(b)

Figure 3. Raman spectra of (a) decorated carbon nanotube and (b) composites.

\subsubsection{X-ray Diffraction (XRD) Analysis}

The crystalline or amorphous nature of the material can be predicted based on XRD profile. The XRD profiles of the $\mathrm{Ti}_{3} \mathrm{AlC}_{2}, \mathrm{Ti}_{3} \mathrm{C}_{2} \mathrm{~T}_{\mathrm{x}}, \mathrm{CNT}, \mathrm{CNTO}$, decorated CNTO, and composites are shown in Figure 4a,b, and drew a $2 \theta$ range between 5 to $90^{\circ}$. The crystalline MAX phase generated sharp peaks at $9.52^{\circ}$ (002), $19.53^{\circ}(004), 34^{\circ}(101), 35.1^{\circ}(102), 36.8^{\circ}(103), 38.99^{\circ}(008), 41.76^{\circ}(104), 42.54^{\circ}(105), 48.48^{\circ}(107)$, $52.36^{\circ}(108), 56.5^{\circ}(109), 60.16^{\circ}(110), 52.36^{\circ}(1011), 64.98^{\circ}(1011), 70.34^{\circ}(1012), 74.02^{\circ}(118)$, and other miscellaneous small peaks [30-36]. Following the etching process, the corresponding MAX phase peaks vanished or shifted, and the sequence of new diffraction peaks was formed. The formed MXene held a crystalline nature and the peak at $7.14^{\circ}(002)$ was a characteristic peak of MXene interplanar crystal space. 
In addition, the peaks originating at $14.36^{\circ}, 19.12^{\circ}, 28.98^{\circ}, 38.86^{\circ}$, and $40.9^{\circ}$ confirmed the crystalline nature of the MXene and attested to the occurrence of etching [32-35]. The peak shift of $\mathrm{Ti}_{3} \mathrm{AlC}_{2}$ from $9.59^{\circ}$ to $6.96^{\circ}$ and the formation of the MXene new peak at $21.57^{\circ}$ indicated that the effective eradication of Al layers occurred (Figure $4 \mathrm{a}$ ). The peak at $38.86^{\circ}$ implied the remains of the layered MAX phase structure without an $\mathrm{Al}$ layer which confirmed the formation of MXene. Further, the separation of the layers after the etching was low; thus, the crystalline nature of the MXene remained the same as the structure of $\mathrm{Ti}_{3} \mathrm{AlC}_{2}$ (Figures $1 \mathrm{f}$ and $4 \mathrm{a}$ ). The carbon nanotubes exhibited a crystalline nature, as confirmed by the $25.88^{\circ}(002), 42.84^{\circ}(100), 43.69^{\circ}(101) 48.94^{\circ}(102)$, and $54.07^{\circ}(004)$ reflection peaks and implied the presence of the concentric cylindrical MWCNT $[48,49]$. In addition, the shifting of the position of $2 \theta$ of the corresponding MWCNT attested to the oxidation of MWCNT and increased the percentage of the sp2 hybridized carbons (Figure 4b) [36-40]. The CFeO generated peaks at $18.36^{\circ}, 30.21^{\circ}, 35.66^{\circ}, 43.33^{\circ}, 53.76^{\circ}$, $57.27^{\circ}, 62.88^{\circ}, 71.37^{\circ}$, and $74.42^{\circ}$, and its corresponding reflection plans were (111), (220), (311), (222), (422), (511), (440), and (533), respectively [50,51]. The CFe generated peaks of zero valent iron nanoparticle at $44.73^{\circ}(110), 64.53^{\circ}(200)$, and $82.39^{\circ}(211)$, and the other peaks were corresponding CNTO signals [52]. The (200) reflection peaks of $\mathrm{CNi}, \mathrm{CCo}$, and $\mathrm{CCu}$ were located at $51.68^{\circ}, 51.68^{\circ}$, and $50.31^{\circ}$, respectively. The (111) peak of CCo and CCu originated at $44.87^{\circ}$ and $43.2^{\circ}$, respectively [53-55]. The new peaks were raised due to the CNTOs and the aggregation of nanoparticles in the CNTOs (Figure $4 b$ ).

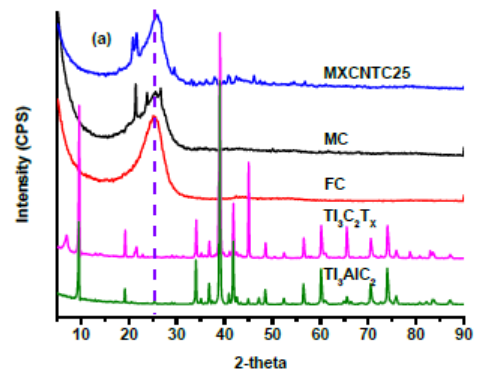

(a)

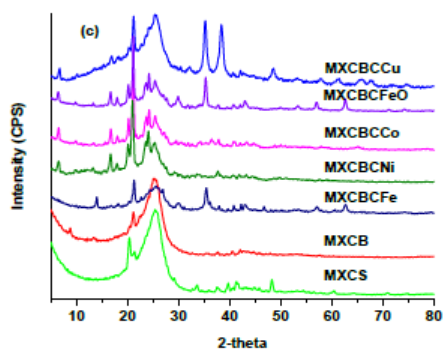

(c)

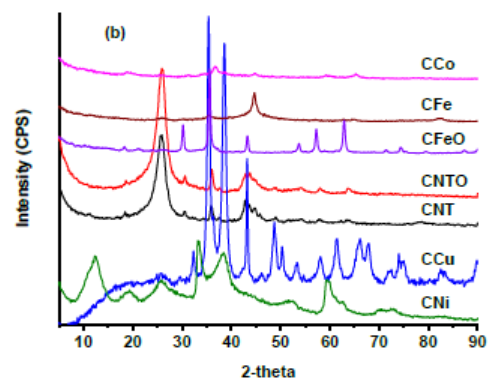

(b)

Figure 4. XRD of (a) MXene, MAX phase, and fabric (b) decorated carbon nanotubes and (c) composites.

The broad peaks of MC exhibited the amorphous nature of the fabric along with the $2 \theta$ peak at $25.52^{\circ}$, which is similar to the peak of carbon nanotubes and confirmed the presence of a graphite structure [5,40]. Further, MC consists of various small peaks which were not above $30^{\circ}$, and all small peaks disappeared with functionalization (Figure 4a). The MXene-fabric composite showed various small peaks, which were absent in FC. Hence, the introduction of the MXene, CNTOs, and nanoparticles generated many small peaks. All of the carbon fabric composites showed distinctive peaks between 20.3-21.24 $4^{\circ}$, and all of the nanocomposites showed characteristic peaks at $6.69^{\circ}$ and $16.82^{\circ}$, except for $\mathrm{MXCBCFe}$, which showed a typical signal at $14^{\circ}$. MXCBCCu, MXCBCFe, and MXCBCFeO exhibited a peak at $35.25^{\circ}$ among the $\mathrm{MXCBCCu}$ generated shoulder peak at $38.46^{\circ}$. Thus, these composites can easily be distinguished from these characteristic peaks and formed from the constitutional elements of the composites. 


\subsubsection{X-ray Photoelectron Spectroscopy Analysis}

The functionalities, surface elemental composition, structure, and bonding nature of the composites can be explained using XPS. The fitting curve of MXene was plotted using overlapping curves of the Gaussian-Lorentzian function and the overlapping curve of the composites was plotted using origin pro. The fitting curves of Ti2p, O1s, and C1s, the F1s of $\mathrm{TI}_{3} \mathrm{C}_{2} \mathrm{~T}_{\mathrm{X}}$, and the C1s of CNTO displayed different peak positions and corresponding functional groups and bonds (Figure 5 and Table S2) [56-58]. The Ti2p fitting curve disclosed five different chemical environments and the corresponding binding energies were 454.5, 456.4, 458.5, 461.3, and $464.5 \mathrm{eV}$. These binding energies indicated the presence of functional groups such as Ti-C $\left(2 \mathrm{p}_{3 / 2}\right), \mathrm{Ti}^{2+}\left(2 \mathrm{p}_{3 / 2}\right), \mathrm{TiO}_{2}\left(2 \mathrm{p}_{3 / 2}\right), \mathrm{Ti}^{2+}\left(2 \mathrm{p}_{1 / 2}\right)$, and $\mathrm{TiO}_{2}\left(2 \mathrm{p}_{1 / 2}\right)$, respectively (Figure $\left.5 \mathrm{a}\right)$. The binding energies positions of the O1s fitting curves such as 529.6, 531.1, 532.3, and 533.8 eV showed corresponding functional groups like $\mathrm{TiO}_{2}, \mathrm{C}-\mathrm{Ti}-\mathrm{Ox}, \mathrm{Al}_{2} \mathrm{O}_{3}$, and $\mathrm{H}_{2} \mathrm{O}$, respectively, (Figure $5 \mathrm{~b}-\mathrm{d}$ ). The presence of $\mathrm{C}-\mathrm{Ti}-\mathrm{Fx}$ generated a single peak at $685.5 \mathrm{eV}$ in the F1s fitting curve (Figure 5c). The C1s fitting curves at 281.1, 283.2, 284.5, and $286.1 \mathrm{eV}$ confirmed the functionalities such as $\mathrm{C}-\mathrm{Ti}-\mathrm{Tx}, \mathrm{C}-\mathrm{C}$, and $\mathrm{CHx}-\mathrm{CO}$. (Figure 5d) [33,35,43,59-62]. Thus, MXene formed with the formula of $\mathrm{Ti}_{3} \mathrm{C}_{2}(\mathrm{OH}, \mathrm{F})$. In the fitting curve of MWCNT, the intense peak at 284.13 $\mathrm{eV}$ was raised due to the $\mathrm{C}-\mathrm{C}$ bond of graphite, while the presence of the oxygen generated weaker peaks between $287-291 \mathrm{eV}$, and its corresponding oxygenic species such as $\mathrm{C}=\mathrm{O}, \mathrm{C}-\mathrm{O}$, and carbonate were triggered. The fitting peak at $285.86 \mathrm{eV}$ confirmed the presence of the defects, which was further backed by the $\mathrm{I}_{\mathrm{D}} / \mathrm{I}_{\mathrm{G}}$ ratio and amount of oxygen $[63,64]$. The defects were comparatively low in CNT and increased by oxidation in CNTO (Figure 5e, Figure S4 and Table S3) [5]. The overlapping curve of XPS exhibited the constitutional elements and its corresponding peak positions. The constitutional elements of MXene were $\mathrm{Cl}, \mathrm{C}, \mathrm{F}, \mathrm{Ti}$, and $\mathrm{O}$, and the corresponding binding energies were 198.7, 285.22, $685.78,459.62$, and $532.65 \mathrm{eV}$, respectively, while those given by N, Co, Ni, Fe, and $\mathrm{Cu}$ were 400.99, $780.1,854.81,710.7$, and $933.65 \mathrm{eV}$, respectively (Figure 5f). In addition, the overlapping curve of the composite lying between $284.3-284.5 \mathrm{eV}$ confirmed that the proper bonding occurred among the constitutional components (Figure S5) [56].

\subsection{Electrical Conductivity (EC) and Surface Properties}

CNT and MXene possess good EC, which is one of the factors influencing the EMI shielding effectiveness $[2,57]$. The electric conductivity and sheet resistance $\left(R_{S}\right)$ of the fabric significantly changed due to the introduction of the MXene, CNTOs, and nanoparticles in the non-woven fabric matrix (Figure 6). The fabricated composite exhibited EC ranging from 12.5 to $2.65 \mathrm{~S} \cdot \mathrm{cm}^{-1}$, and $\mathrm{R}_{\mathrm{s}}$ lay between 13.98 and $2.08 \Omega \cdot \mathrm{sq}^{-1}$. The MXCNTNiC25 hit a maximum $\mathrm{R}_{\mathrm{s}}$ of $13.98 \Omega \cdot \mathrm{sq}^{-1}$ and a minimum conductivity of $2.65 \mathrm{~S} \cdot \mathrm{cm}^{-1}$, which was due to the high surface defect (Figure 21 ). The functionalization process increased EC by $10.1 \%$ and changed $\mathrm{R}_{\mathrm{S}}$ by $20.9 \%$. Thus, the functionalization process minimized the defect and increased the electron mobility. The filtration-based composite showed EC above $10 \mathrm{~S} \cdot \mathrm{cm}^{-1}$ while MXCBCFeO and MXCBCFe exhibited a maximum and minimum of 12.5 and $8.81 \mathrm{~S} \cdot \mathrm{cm}^{-1}$, respectively. The EC of MXCB, MXCBCNi, MXCBCCo, MXCS, and MXCBCCu were 12.1, 11.3, 11.65, 11.8, and $11.22 \mathrm{~S} \cdot \mathrm{cm}^{-1}$, respectively, whereas the corresponding $R_{\mathrm{S}}$ were $2.08,2.38,2.11,2.2$, and $2.56 \Omega \cdot \mathrm{sq}^{-1}$, respectively. Spray coated MXene-CNTO composites displayed EC below $10 \mathrm{~S} \cdot \mathrm{cm}^{-1}$, among which MXCNTC30 exhibited $9.55 \mathrm{~S} \cdot \mathrm{cm}^{-1}$. Hence, $14.62 \%$ of electric conductivity was increased by introducing MXene, CNTOs, and decorated carbon nanotubes into the MC network (Figure 6, Figure S6 and Table S6). The MXene has a high instinct electric conductivity due to its metal like nature [5]. In addition, the doping of CNT by nitrogen expands the EC while the degree of oxidation reduces the conductivity. However, the MXene-carbon fabric composite showed a maximum of $8.84 \mathrm{~S} \cdot \mathrm{cm}^{-1} \mathrm{EC}$ while the CNTO-carbon fabric composite displayed $16.32 \mathrm{~S} \cdot \mathrm{cm}^{-1}$ of EC. Thus, MXene-CNTO-nanocomposite showed conductivity between the MXene and CNTO composites $[2,5,24,65]$. These defects limit the electron mobility and electron hopping along the fiber $[66,67]$. We attempted to explain this using the hydrophobic nature of the composites. The wetting ability of the surface can be explained based on the contact angle, which above $90^{\circ}$ is called a water-repellent surface, while below $90^{\circ}$ is considered a water loving surface. Further, 
the contact angle is dependent on the surface roughness and energy. Roughness increases the defect, thus increasing the surface energy and surface roughness raise the hydrophobic nature $[5,16,56]$. The contact angle, wetting energy, spreading coefficient, and work of adhesion of MXCNTNiC25 were $131.3^{\circ}$, $-48.09 \mathrm{mN} \cdot \mathrm{m}^{-1},-120.89 \mathrm{mN} \cdot \mathrm{m}^{-1}$, and, $24.71 \mathrm{mN} \cdot \mathrm{m}^{-1}$, respectively, while FC exhibited $134.18 \mathrm{mN} \cdot \mathrm{m}^{-1}$, $-50.7 \mathrm{mN} \cdot \mathrm{m}^{-1},-123.54 \mathrm{mN} \cdot \mathrm{m}^{-1}$, and $22.06 \mathrm{mN} \cdot \mathrm{m}^{-1}$, respectively. In addition, the other composites showed no coated angle that was due to the absorption of the water or well spread on the surface of the composites. The conductivities of most of the composites were high considering the fewer surface defects while the conductivity of the MXCNTNiC25 showed the lowest conductivity, confirming that MXCNTNiC25 possessed high defect and surface roughness (Figures 21 and 6, and Figure S7).

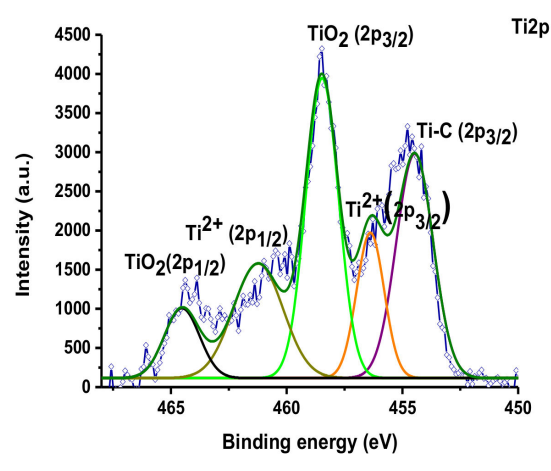

(a)

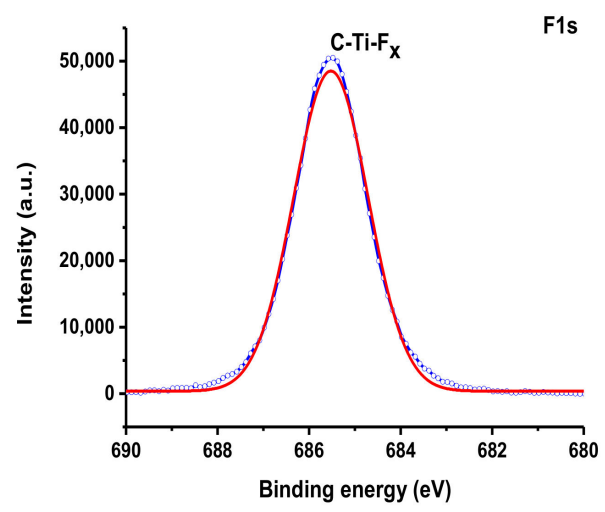

(c)

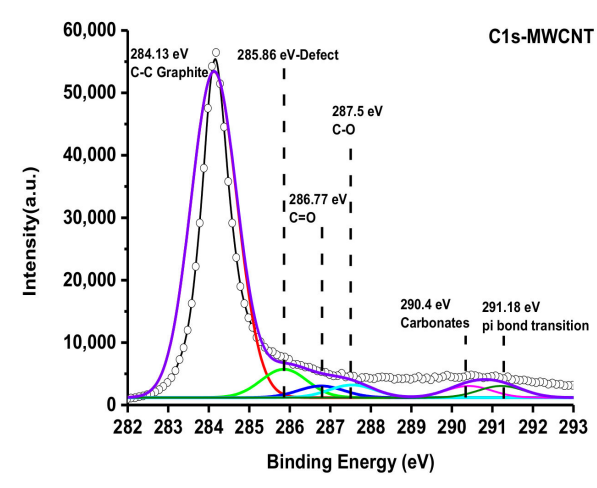

(e)

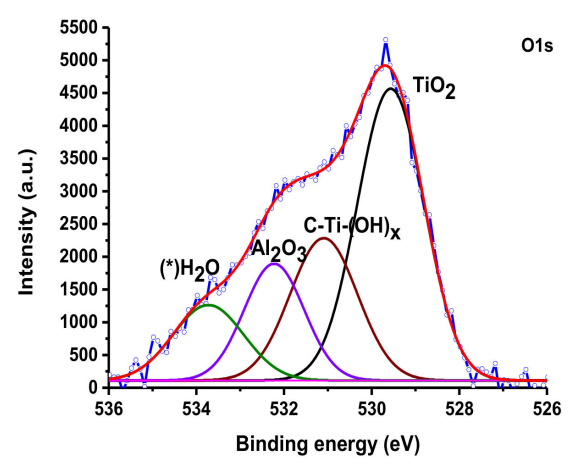

(b)

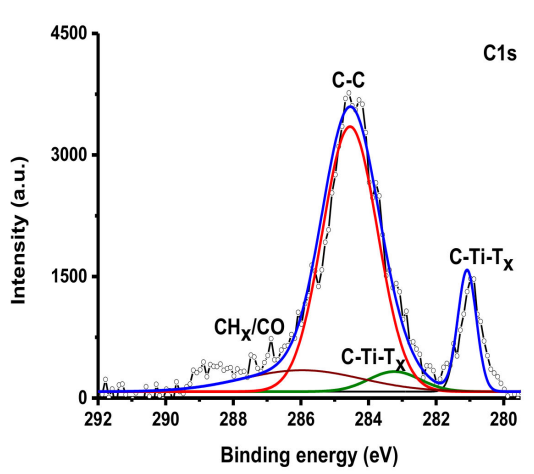

(d)

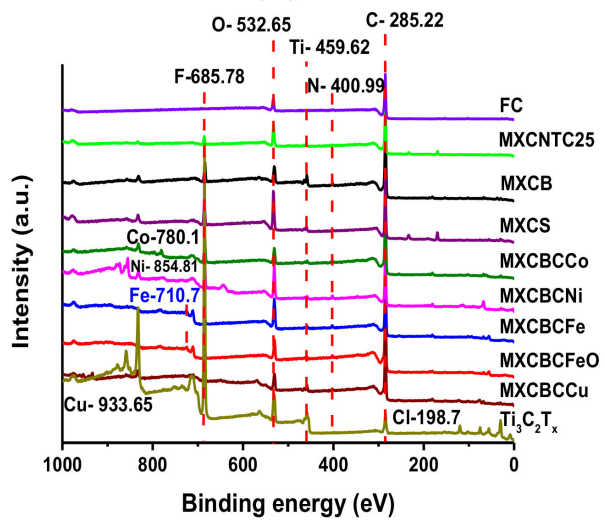

(f)

Figure 5. XPS fitting curves of (a) Ti2p, (b) O1s, (c) C1s, (d) F1s, (e) C1s of MWCNT, and (f) survey of the composites. 


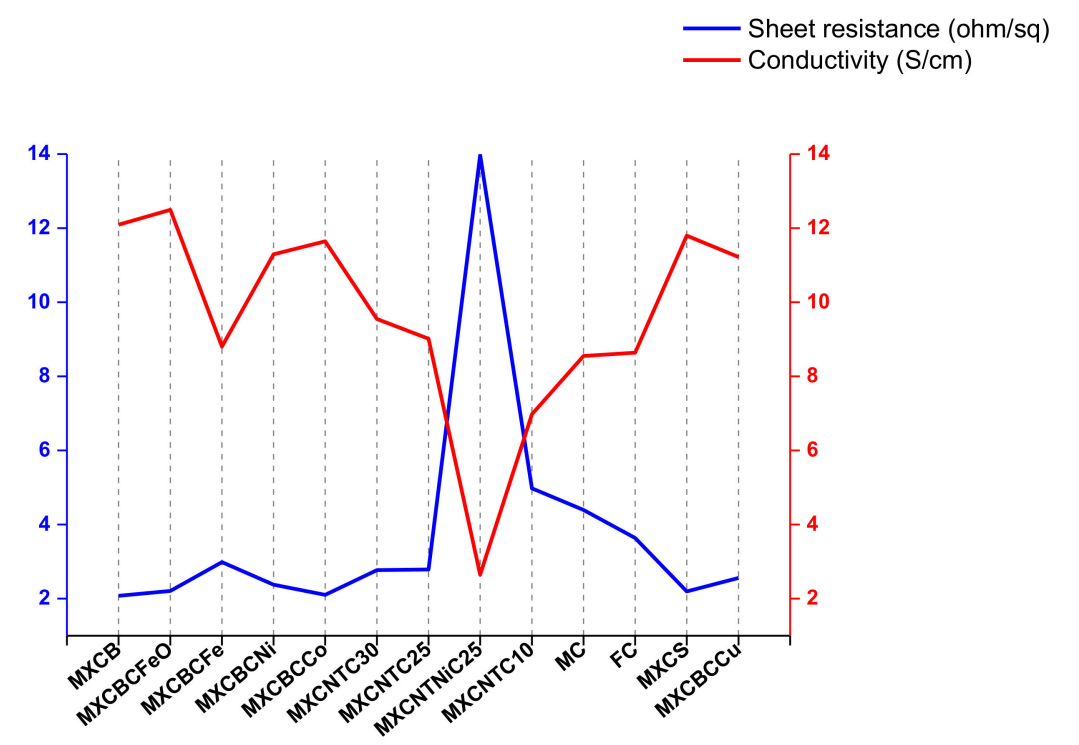

Figure 6. Electric conductivities and sheet resistances of the composites.

\subsection{Magnetic Properties of the Composites}

The magnetic properties of the composites at $300 \mathrm{~K}$ were studied using hysteresis loop measured at $300 \mathrm{~K}$ (Figure 7 and Figure S8). The saturation magnetization (Ms), remanence (Mr), coercivity (Hc), and coefficient of squareness of hysteresis loops $(\mathrm{Kp})$ can be determined using hysteresis loop [68-71]. In addition, the $\mathrm{Kp}$ can be calculated using the $\mathrm{Mr} / \mathrm{Ms}$ ratio. Alborzi et al. and co-workers showed that decreasing the Kp value enhanced the super magnetic property, whereas diminishing the particle size decreased the Hc and increased the Ms. In addition, super magnetic material can be created by minimizing the Hc [69]. The formation of a cluster structure improves the Ms and dropping the cluster size leads to lower magnetic energy and super magnetic behavior [71]. Thus, the magnetic properties of the materials were influenced by various factors such as the geometry, size, functional groups, morphology, and crystallinity [72]. Further, precursor salt also affects the magnetic properties of the material and the synthesis of $\mathrm{Fe}_{3} \mathrm{O}_{4}$ by using ferrous and ferric sulfate generates $46.7 \mathrm{emu} \cdot \mathrm{g}^{-1}$ of Ms while ferrous and ferric chloride produce $55.4 \mathrm{emu} \cdot \mathrm{g}^{-1}$ of Ms and the magnetization of bulk $\mathrm{Fe}_{3} \mathrm{O}_{4}$ is $93 \mathrm{emu} \cdot \mathrm{g}^{-1}$ [69]. All the composites possessed nonlinear behavior against applied field and showed the hysteresis loop (Figure 7 and Figure S8). The Ms of the composites ranged from $0.45-0.009 \mathrm{emu} \cdot \mathrm{g}^{-1}$ and the saturated magnetic strength was placed between 9.95-3284.4 Oe. The Kp ranging from 0.022 to 1.128 and $\mathrm{Mr}$ fluctuated between $2.2 \times 10^{-4}-0.187 \mathrm{emu} \cdot \mathrm{g}^{-1}$, whereas a $1.27-232.3$ Oe range of Hc was given by the composites (Figure 7, Figure S8 and Table 1). According to Figure 7b, the magnetization of the FC approached almost zero, compared with other composites, and the hysteresis loop was not smooth, like other composites. It was considered to be due to the irregular arrangement of the fibers (Figure S8c and Figure 2a), and a study by Lu et al. showed that carbon fibers are non-magnetic materials. Thus, functionalization induced the magnetic behavior of the MC [7]. Furthermore, the Ms, Mr, and Kp values were lower than those of the others. This confirmed that interconnecting fibers using nanomaterials alters the magnetic property of the nonwoven fabric (Table 1 and Figure 2b-1). MXCBCNi and MXCBCCo behaved differently when applied magnetic field increased the magnetization and also increased while others exhibited constant magnetization (Figure $7 \mathrm{~b}$ ). A part of the loop of MXCBCFe pass through the origin, did not have negative $\mathrm{Mr}$ and positive $\mathrm{Hc}$ value, and had more than one loop, whereas $\mathrm{MXCBCFeO}$ did not have a positive Hc value, and instead the loop was located near the Mr point. Further, $\mathrm{MXCBCFeO}$ and $\mathrm{MXCBCFe}$ possessed high Kp values of 1.128 and 0.095 , where 0.095 was lowest among all composites. Additionally, $\mathrm{MXCBCFeO}$ and $\mathrm{MXCBCFe}$ contained the lowest Ms, Hc, and saturated magnetic strength, which 
affect the EMI shielding of the nanoparticle-based composites. According to this study, increasing Kp increased the EMI shielding.

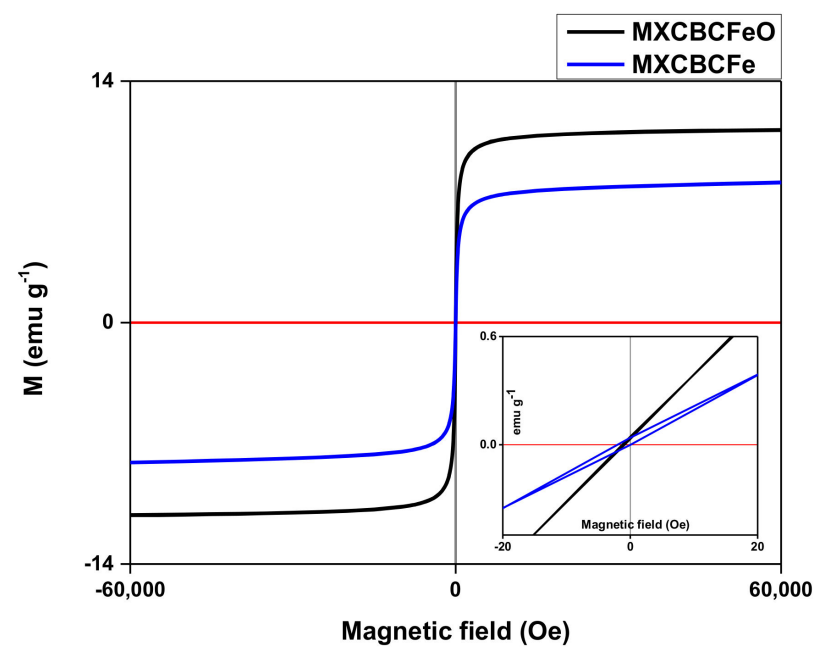

(a)

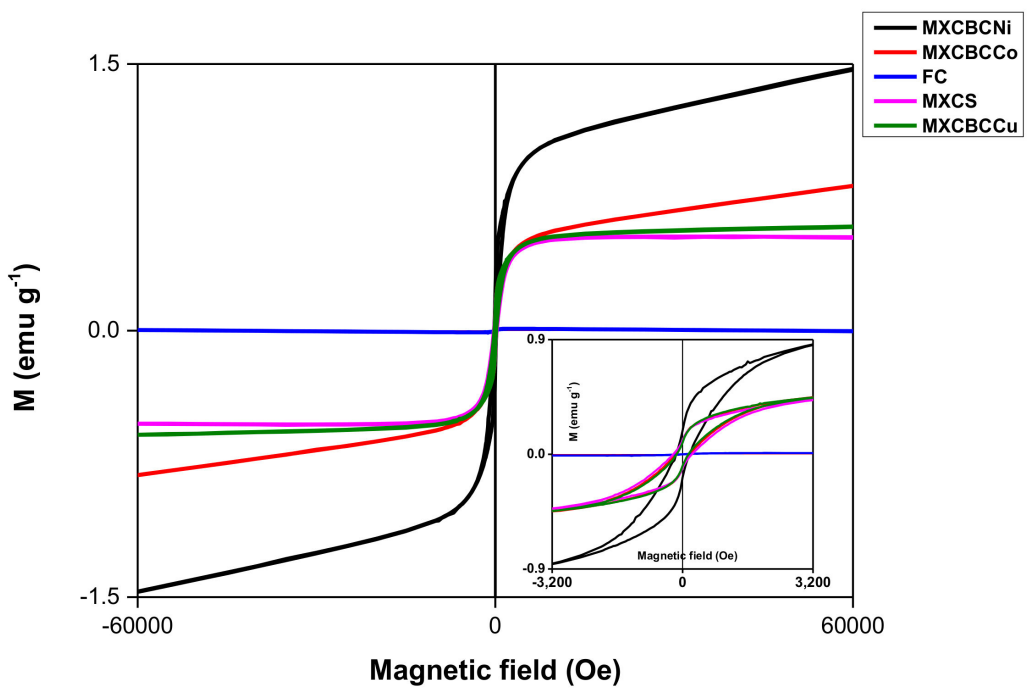

(b)

Figure 7. Magnetization against applied field at $300 \mathrm{~K}$ : (a) Fe- and $\mathrm{Fe}_{3} \mathrm{O}_{4}$-based composites and (b) Ni, $\mathrm{Co}, \mathrm{Cu}$, and non-nanoparticle-based composites.

Table 1. Comparison of saturation magnetization, retentivity, and coercivity of the composites.

\begin{tabular}{|c|c|c|c|c|c|}
\hline Composites & $\begin{array}{l}\text { Saturated Magnetic } \\
\text { Strength (Oe) }\end{array}$ & $\begin{array}{c}\text { Saturation } \\
\text { Magnetization (Ms) } \\
(\mathrm{emu} \cdot \mathrm{g}-1)\end{array}$ & $\begin{array}{l}\text { Remanence (Mr) } \\
\quad(\mathrm{emu} \cdot \mathrm{g}-1)\end{array}$ & $\begin{array}{l}\text { Coefficient of Squareness of } \\
\text { Hysteresis Loops }(\mathrm{Kp})=\mathrm{Mr} / \mathrm{Ms} \\
\text { (Dimensionless) }\end{array}$ & $\begin{array}{l}\text { Coercivity } \\
\text { (Hc) (Oe) }\end{array}$ \\
\hline MXCВCCu & 3284.4 & 0.45 & 0.08 & 0.178 & 144.59 \\
\hline MXCS & 3200 & 0.43 & 0.1 & 0.233 & 232.3 \\
\hline $\mathrm{FC}$ & 2587.7 & 0.0099 & $2.2 \times 10-4$ & 0.022 & 22.34 \\
\hline МХСВССо & 3195.9 & 0.45 & 0.096 & 0.213 & 185.57 \\
\hline MXCBCNi & 3184.9 & 0.86 & 0.187 & 0.217 & 152.58 \\
\hline MXCBCFe & 21.4 & 0.41 & 0.039 & 0.095 & 2.02 \\
\hline $\mathrm{MXCBCFeO}$ & 9.95 & 0.39 & 0.44 & 1.128 & 1.27 \\
\hline
\end{tabular}




\subsection{Electromagnetic Shielding Effectiveness (EMI-SE) of Composites}

The study employed filtration and spray coating techniques, for which $30 \mathrm{~g} \cdot \mathrm{m}^{-2}$ of areal density fabrics were used, while Ni coated fabric was $20 \mathrm{~g} \cdot \mathrm{m}^{-2}$ of areal density. For the spray coating, MXene colloidal solution and dispersed CNTO and SDS solution were used, while each coating used $40 \mathrm{~mL}$ of solution. A maximum of $100 \mathrm{~mL}$ of colloidal solution was used in the filtration process. In spray coating composites, one MXene layer was sandwiched between two layers of CNTO, while one MXene layer placed between CNTO and nanoparticles decorated CNTO in filtrated composites. For the spray coating process, SDS was used as surfactant and CTAB was used to disperse nanoparticles decorated carbon nanotube. The spraying and filtration process changed the thickness and pore size of the fabric. The EMI shielding was performed in the region of the $\mathrm{X}$ band and $\mathrm{S}$ band. $\mathrm{X}$ band $(8-12.4 \mathrm{GHz})$ EMI-SE was performed for all composite, while $S$ band (1-3 GHz) EMI-SE was only measured for spray coated composites (Figure 7a). The intercalation of nanomaterials with FC and MC significantly increased the EMI shielding. The spray coated composite showed a higher EMI-SE between 1.5 to $2.6 \mathrm{GHz}$ and non-woven carbon fabric preferable over Ni coated carbon fabric as it showed a lower EMI-SE than the other composites (Figure 8a,b). The non-fabrics were flexible and contained physically interconnected fibers with a three-dimensional reticular structure. When the incident wave hit the surface of the shielding materials, the reflection, multiple reflection, absorption, and transmission occurred. The strength of this mechanism varies based on the materials used for EMI-SE. According to the Simon formalism, EMI-SE depends on the electric conductivity and thickness of the material, and the length of the carbon fiber has no influence on EMI shielding. Further, hiking areal and volume density increase the electric conductivity and EMI shielding. A study by Lu et al. showed that the EMI-SE of $50 \mathrm{~g} \cdot \mathrm{m}^{-2}$ of carbon fabric is $30.2 \mathrm{~dB}$, while $30 \mathrm{~g} \cdot \mathrm{m}^{-2}$ produces $23.1 \mathrm{~dB}$, and the EMI shielding of carbon fabric is independent of the frequency range $[7,73,74]$. Hence, increasing the areal density of the carbon fabric increases the EMI-SE. The $30 \mathrm{~g} \cdot \mathrm{m}^{-2}$ of MC gave rise to a maximum of $28.5 \mathrm{~dB}$ of EMI-SE in the S-band whereas $31.7 \mathrm{~dB}$ of EMI-SE was generated in the X-band region, and this was further enhanced by functionalization up to $43.9 \mathrm{~dB}$ of maximum (Table $\mathrm{S} 5$ and Figure $8 \mathrm{a}, \mathrm{b}$ ). This result was almost consistent with that of the Lu et al. study that EMI-SE was independent of frequency. This is despite the fact that the areal density and electric conductivity is low while functionalization had a greater effect on EMI-SE. After the functionalization, the MC possessing the magnetic property was advanced criteria for the high EMI-SE.

All the FC-based nanocomposites' minimum, maximum, and averaged shielding were above $99.99 \%$ of incident wave in the X-band region while spray coated composites' maximum shielding was just below $99.99 \%$ and the minimum and average laid between $99-99.9 \%$. For the FC-based composite of $\mathrm{SE}$, reflection $\left(\mathrm{SE}_{\mathrm{R}}\right)$ and absorption $\left(\mathrm{SE}_{\mathrm{A}}\right)$ prevented $90 \%$ and $99.9 \%$, respectively. MXCNTC30 and MXCNTC25 displayed $99.99 \%$ of maximum, minimum, and average shielding whereas the others showed $99.9 \%$ of shielding in the X-band region. In addition, spray coated composites $\mathrm{SE}_{\mathrm{A}}$ were in the range of $99-99.9 \%$ and $90 \%$ of reflection. The spray coated composite showed maximum shielding of $99.9 \%$ (Figure $8 \mathrm{a}-\mathrm{d}$ and Table S5). The maximum EMI-SE shown by MXCNTC30, MXCNTC25, MXCNTNiC25, MXCNTC10, and MC in the $S$ band were 39.6, 39.9, 34.1, 33.2, and $28.5 \mathrm{~dB}$, respectively, whereas $47.3,47.1,34.9,39.9$, and $39.6 \mathrm{~dB}$ were given in the X-band region. Thus, the shielding of composites comparatively increased when the measurement frequency reached from the $\mathrm{S}$ band to the $\mathrm{X}$ band. Further, it was obvious that above 25 coating cycles, the shielding of composite was significantly reduced in the S-band region. It was considered that increasing the amount of MXene and CNTO reduced the conductivity and shielding ability of composite in the S band. When MXCNTC30 went from the $S$ band to the $X$ band, the EMI-SE increased by $19.4 \%$, while it increased by $18 \%$ for MXCNTC25. (Figure 7a and Table S5). Thus, EMI-SE for nanocomposites were studied in the $\mathrm{X}$-band region. By contrast, MXCNTNiC25 showed the lowest shielding in the $\mathrm{S}$ and $\mathrm{X}$ bands, due to surface defects and low electric conductivity. The defect was due to the reduced adhesive ability of MXene-CNTO composites with Ni coated carbon fabric, and the carbon fabric showed low defects (Figures 21 and 8 and Figure S3f). The MXCNTNiC25 exhibited higher EMI shielding than MC as 
the porosity was diminished. Nonetheless, all the coated carbon fabric showed higher EMI shielding than MXCNTNiC25 [1-3]. The maximum EMI-SE of MXCB, MXCBCFeO, MXCBCFe, MXCBCNi, MXCBCCo, MXCBCCu, and MXCS were 47.6, 45.9, 46.7, 45, 46, 43.6, and $50.5 \mathrm{~dB}$, respectively. The nanoparticle-free composites displayed higher blocking ability than others and the mushroom gills like structure significantly reduced the EMI-SE as compared with FC, whereas the coral like structure showed a higher EMI-SE than FC (Figures $2 g-i$ and $8 b$ and Table S5). Despite this, all showed above $45 \mathrm{~dB}$, except for MXCBCCu (Figure 7 and Table S5). The absorption was dominant over reflection, and reflection stayed nearly constant for all composites. Thus, the changing of the shielding effectiveness was due to the absorption of the composites. The composite showed electric conductivity in the range of 2-13 S. $\mathrm{cm}^{-1}$, which was not sufficient to produce good $\mathrm{SE}_{\mathrm{R}}$. The effective percolation of the conductive network facilitates the electron mobility and reflection and enhances the ohmic loss [1-3,7]. The intercalation of the MXene, CNTO, and nanoparticle decorated CNTO increase the electric conductivity, though the lack of an effective conductive network minimizes the $\mathrm{SE}_{\mathrm{R}}$ [7]. In this case, part of the incident wave was reflected, while most of the remaining part underwent absorption and multiple reflection. The maximum $\mathrm{SE}_{\mathrm{A}}$ of $\mathrm{MXCB}, \mathrm{MXCBCFeO}, \mathrm{MXCBCFe}, \mathrm{MXCBCCo}$, MXCS, MXCNTC30, and MXCNTC25, were all above $33 \mathrm{~dB}$, while the $\mathrm{SE}_{\mathrm{R}}$ of all the composites were above $10 \mathrm{~dB}$ (Figure $8 \mathrm{c}, \mathrm{d}$ and Table S5). In addition, the Kp value above 0.09 led to shielding above $99.99 \%$ (Figure 7a,b and Table 1). Thus, absorption can be achieved by different internal geometry, functionalization, ohmic loss, defects, and magnetic property of materials. In addition, the CNTO contributed inner tube scattering leads to a higher $\mathrm{SE}_{\mathrm{A}}$ because the blend of different components establishes diverse phases which enhance multiple reflection and absorption [1]. The functionalization considerably increased the specific shielding effectiveness (SSE) and absolute effectiveness (SSE/ $t$ ), and the SSE and SSE/t of the FC were $401.93 \mathrm{~dB} \mathrm{~cm} \mathrm{~cm}^{-3} \cdot \mathrm{g}^{-1}$ and $12639.95 \mathrm{~dB} \mathrm{~cm}^{-2} \cdot \mathrm{g}^{-1}$, respectively. The intercalations of zero, one-, and two-dimensional materials in the three-dimensional fiber network significantly reduced the SSE and SSE/t. Further, iron-based composited displayed lower SSE and comparatively lower SSE/t (Figure 8e and Table S6).

Figure $8 \mathrm{f}$ and Table S4 compared the EMI-SE with previous corresponding work. The dip coated CNTO non-woven fabric (basic weight $20 \mathrm{~g} \cdot \mathrm{m}^{-2}$ ) showed a maximum of $33 \mathrm{~dB}$, which is substantially lower than that of the MXene-CNTO-nanoparticle composite [5]. The polystyrene-MWCNT composite showed a maximum EMI-SE of about $22 \mathrm{~dB}$. The Polyvinylidene fluoride (PVDF)-MWCNT composite exhibited $28.5 \mathrm{~dB}$ of EMI-SE with a thickness of $0.2 \mathrm{~cm}$ while the segregated carbon nanotube-polypropylene composite showed an EMI-SE of $48.3 \mathrm{~dB}$ with $0.22 \mathrm{~cm}$ of thickness and interconnected MWCNT polymeric matrix exhibited $27 \mathrm{~dB}$ at $18 \mathrm{GHz}[1,12,13,74,75]$. In addition, the spongy carbon nanotube composite disclosed 54.8 with thickness of $0.18 \mathrm{~cm}$ in the X-band region [16]. Most of the study showed the EMI shielding range of the CNT composite was 20-30 dB with higher thickness. The different types of fillers and geometry altered the shielding ability of the MWCNT. Thus, we analyzed various combinations of materials with different geometries in order to increase the shielding effectiveness of the non-woven fabric and MWCNT. MXene film crammed between Polyethylene terephthalate polymer film showed an excellent EMI shielding of $92 \mathrm{~dB}$ with $4600 \mathrm{~S} \cdot \mathrm{cm}^{-1}$ electrical conductivity [2]. Cao et al. and coworkers highlighted that nacre-like MXene-cellulose nanofiber composite showed an EMI shielding range of 5.3-25.8 dB based on the percentage of MXene added to one dimensional cellulose fiber [76]. The MXene-carbon fabric composite exhibited an EMI-SE of $43.2 \mathrm{~dB}$ [24]. Hence, a combination of the MXene-CNTO-based composite dramatically increased the EMI-SE. Further, the carbon fabric-based composite showed lower shielding; therefore, the manufactured composite was considered to have excellent EMI-SE ability (Figure 8f). 


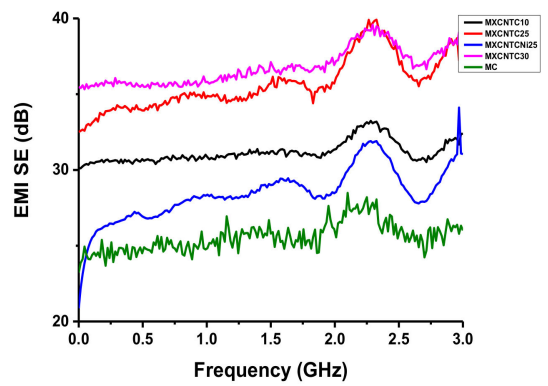

(a)

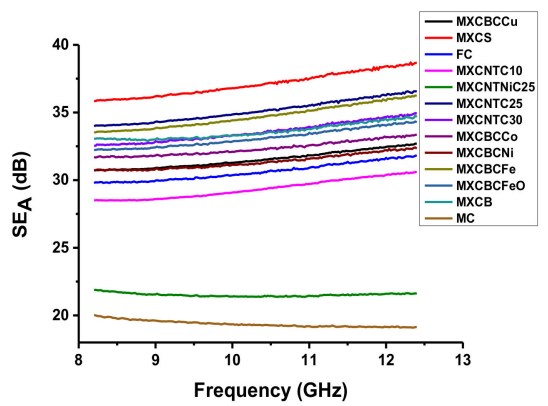

(c)

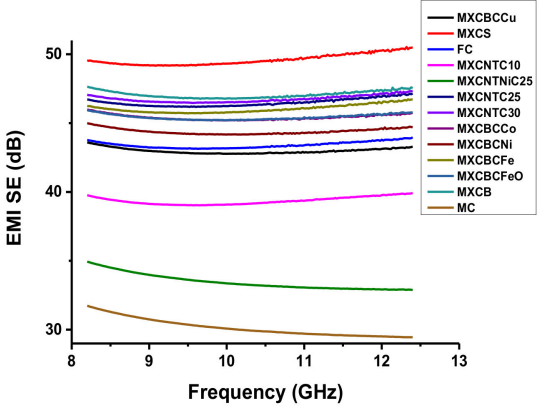

(b)

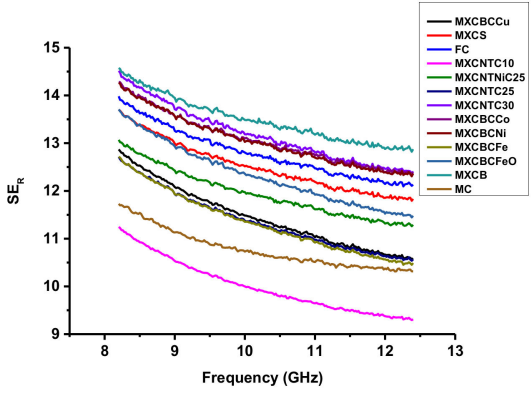

(d)

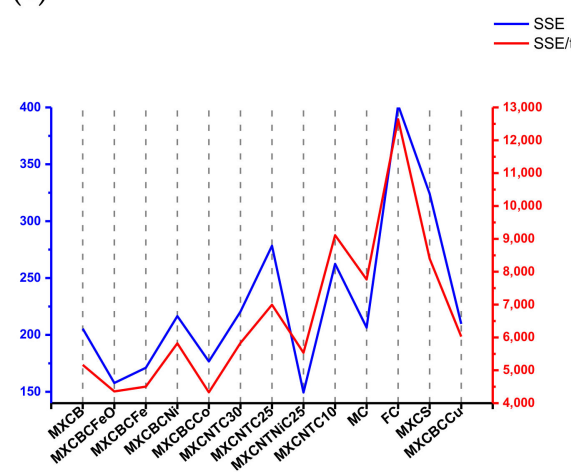

(e)

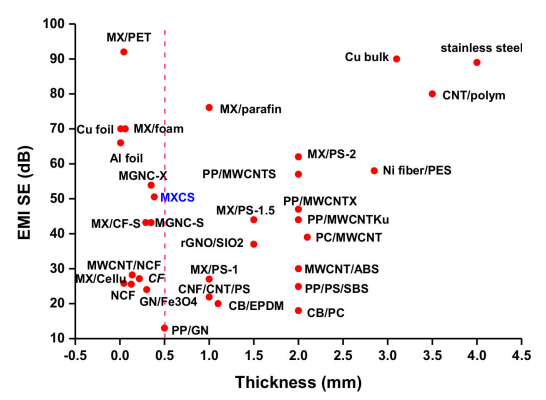

(f)

Figure 8. Electromagnetic interference (EMI) shielding of the composites: (a) Total EMI shielding (S band), (b) total EMI shielding (X band), (c) absorption (X band), (d) reflection (X band), (e) specific shielding effectiveness (SSE) and absolute effectiveness (SSE/t) of the composites, and (f) EMI shielding comparison of different composites.

\subsection{Thermal Stability and Thermo Gravimetric Analysis of Composites.}

Thermogravimetric analysis (TGA) and differential thermal analysis (DTA) were used to investigate the thermal stabilities of the composites. TGA graphs were plotted in the temperature range of $30-1000{ }^{\circ} \mathrm{C}$ while DTA graphs were plotted in the range of $30-900{ }^{\circ} \mathrm{C}$ (Figure 9a-d). The TGA 
and DTA analysis were carried out using $\mathrm{Al}_{2} \mathrm{O}_{3}$ crucible in a nitrogen environment with a heating rate of $10{ }^{\circ} \mathrm{C} \cdot \mathrm{min}^{-1}$. The composites exhibited an outstanding thermal stability over $100{ }^{\circ} \mathrm{C}$. Most of the composites had a starting point of degradation above $140^{\circ} \mathrm{C}$, and the degradation beginning lay between $120-255{ }^{\circ} \mathrm{C}$, where MXCNTC30 showed $120{ }^{\circ} \mathrm{C}$. In addition, MXCB, MXCBCFeO, $\mathrm{MXCBCFe}, \mathrm{MXCBCNi}, \mathrm{MXCBCCo}$, and MXCNTNiC25 prohibited degradation above $150^{\circ} \mathrm{C}$ among mushroom gill like $\mathrm{MXCBCCu}$ decomposed at lower temperature of $127.5^{\circ} \mathrm{C}$, whereas the degradation temperatures of the other composites were below $149^{\circ} \mathrm{C}$ and above $127.5^{\circ} \mathrm{C}$ (except MXCNTC30 at $120^{\circ} \mathrm{C}$ ). The rapid mass changing percentage of composites occurred between $120-897.5^{\circ} \mathrm{C}$ where spray coated composite hit the higher value (above $700^{\circ} \mathrm{C}$ ), while filtered gave rise below $653^{\circ} \mathrm{C}$ (Figures $2 \mathrm{i}$ and $9 \mathrm{a}, \mathrm{c}$ and Table S7). The temperature between the $30-1000{ }^{\circ} \mathrm{C}$ range of weight losing percentage of the composites were $6-36 \%$, in which $\mathrm{MXCBCFeO}$ gave a maximum of $35.71 \%$ while $8.85 \%$ of weight loss occurred in MXCNTC25 (Figure 9a,c and Table S7).The MC and FC degradations of the whole temperature range were $6.84 \%$ and $6.4 \%$, respectively, and the corresponding degradation beginning temperatures were $190{ }^{\circ} \mathrm{C}$ and $255.5^{\circ} \mathrm{C}$, respectively (Figure $9 \mathrm{a}, \mathrm{c}$ and Table S7). The functionalization of MC by using chitiooligosaccharide increased the thermal stability. This was attributed to the fact that the chitiooligosaccharide contains lots of the hydroxyl groups, thus creating the hydrogen bonding which led to higher thermal stability [68]. According to Raagulan et al., rapid mass changes occurred due to the loss of surface functionalities, water, and decomposition of fabric $[5,6,63,64]$. In addition, the MXene-carbon fabric composite prohibited degradation up to $235^{\circ} \mathrm{C}$ and a Gamage et al. study showed CNTO-carbon fabric composites prohibited degradation until $284^{\circ} \mathrm{C}$. The combination of MXene, CNTO, and nanoparticles considerably diminished the degradation temperature (Table S7). The DTA analysis revealed various peaks raised due the degradation of the composites (Figure 9b,d). Strong peaks were placed in the region of the rapid changing of TGA curve [77]. Further, we observed that the slope of the TGA curve depends on the intensity of the corresponding peaks in DTA analysis, and that the high intensity of the DTA peaks lead to rapid degradation. The more intense peaks were located between $209-934.2{ }^{\circ} \mathrm{C}$ temperature range (Figure $9 \mathrm{~b}, \mathrm{~d}$, Figure S9a-m and Table S8). It was obvious that the introduction of the nanomaterials in the fabric network shifted the peaks to lower temperatures and diminished the decomposition temperature.

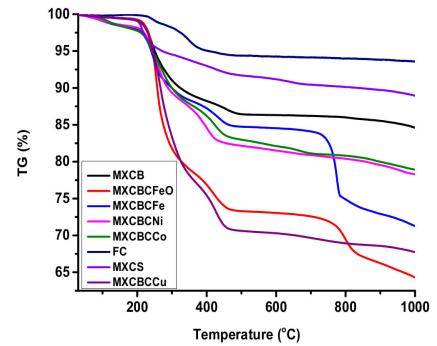

(a)

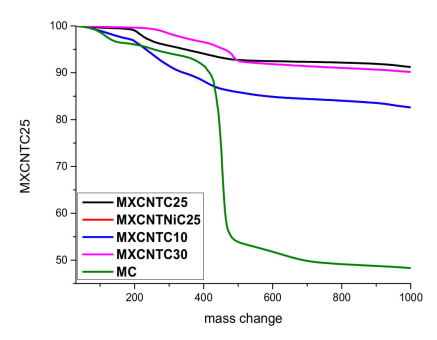

(c)

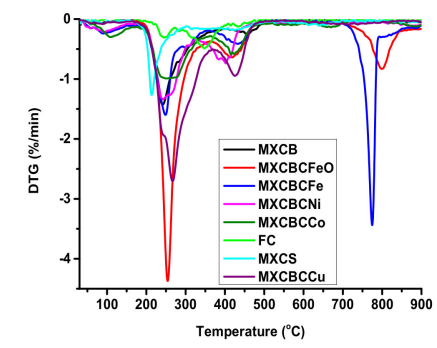

(b)

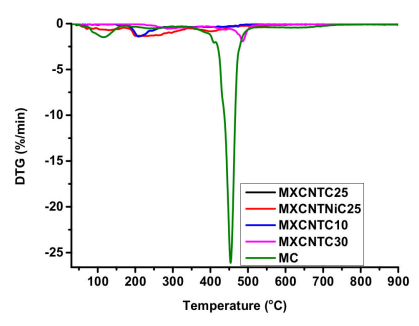

(d)

Figure 9. TGA and differential thermal analysis (DTA) analysis of the composites (a) TGA of filtration-based composite, (b) TGA of filtration-based composite, (c) TGA of spray coated composite, and (d) DTA of spray coated composite. 


\section{Conclusions}

The filtration-based nanocomposite and spray coated nanocomposite were successfully prepared. The effect of the functionalization of non-woven carbon fabric was analyzed as well. The composites were lightweight and thinner. The density of the composites ranged from 0.108 to $0.288 \mathrm{~g} \cdot \mathrm{cm}^{-3}$ while the thickness of the composites ranged between $0.266-0.408 \mathrm{~mm}$. The ranges of $\mathrm{Ms}$ and Mr were $0.0099-0.86 \mathrm{emu} \cdot \mathrm{g}^{-1}$ and $0.00022-0.44 \mathrm{emu} \cdot \mathrm{g}^{-1}$, respectively. The MXCBCNi displayed a higher Ms of $0.86 \mathrm{emu} \cdot \mathrm{g}^{-1}$ and a $0.44 \mathrm{emu} \cdot \mathrm{g}^{-1}$ of $\mathrm{Mr}$ was shown by MXCBCFeO. The MXCCFeO exhibited a maximum Kp of 1.128 while FC was 0.022 . The functionalized carbon fabric displayed a hydrophobic nature with a $134.18^{\circ}$ contact angle, whereas MXCNTC25 displayed a contact angle of $131.3^{\circ}$. The electric conductivities of the composites varied between $2.65-12 \mathrm{~S} / \mathrm{cm}$, whereas the surface resistances were in the range of 2.08-13.98 $\Omega$ /sq. The composites showed good thermal stability and resisted complete thermal degradation above $120^{\circ}$. In addition, functionalization increased the thermal stability and prevented degradation up to $255.5^{\circ}$. The maximum EMI-SE shown by MXCS was $50.5 \mathrm{~dB}$, and the EMI shielding range of the composite was 50.5-28.5 dB. The maximum $\mathrm{SE}_{\mathrm{R}}$ and $\mathrm{SE}_{\mathrm{A}}$ were shown by MXCB and MXCS, respectively. The ranges of SSE and SSE/t were 149.37-401.93 dB $\mathrm{cm}^{3} \cdot \mathrm{g}^{-1}$ and $4330.82-12,639.35 \mathrm{~dB} \mathrm{~cm}{ }^{2} \cdot \mathrm{g}^{-1}$, respectively, where FC showed maximums of both. Hence, the manufactured fabric composite presented high EMI shielding, magnetic behavior, low density, smaller thickness, and flexibility.

Supplementary Materials: The following are available online at http:/ / www.mdpi.com/2079-4991/9/4/519/s1, Figure S1: SEM image of CNTO decorated by (a) Fe3O4 (b) Fe (c) Ni (d) Cu; Figure S2: SEM image of carbon fabric composite of (a) MC (500) (b) MXCB (500) (c) surface of MXCS (10000) (d) MXCBCFeO (500) (e)MXCBCFe (500) (f)MXCBCNi (450) (g) MXCBCCo (150) (h) MXCBCCo (100000) (i) MXXBCCu (45000) (j) MXCNTC30 (100) (k) MXCNTNi25 (1000) (l) MXCNT10 (400); Figure S3: SEM image of (a) MC $(\times 500),(b)$ MC $(\times 2000)$, (c) cracks on fiber $(\times 300000)$, (d) MXene-CNTO coated carbon fabric $(\times 500)$, (e) MXene-CNTO coated carbon fabric $(\times 300)$, (f) MXene-CNTO coated fabric (Ni coated fabric) $(\times 300)$, (g) MXene and CNTO on the surface of the fabric $(\times 120000)$, (h) Ti3AlC2 (×22000), (i) Ti3C2Tx (×50000) and (j) Ti3C2Tx (×80000); Figure S4: Normalized curve of Raman spectrum of (a) decorated CNT, (b) composites (c) CNT, (d) CNTO, (e) CCu, (f) CCo, (g) CNi, (h) CFe, (i) $\mathrm{CFeO}$, (j) FC (k) comparison of MXene, MAX phase and MC, (l) MC, (m) MXCS, (n) MXCBCCu, (o) MXCBCCo, (p) MXCBCNi, (q) MXCBCFe, (r) MXCBCFeO and (s) MXCB; Figure S5: The c1s fitting curve of the MXene, functionalized fabric and composites; Figure S6: Resistivity profile of the composites; Figure S7: Contact angle of (a) MXCNTNiC25 (b) FC and (c) represent other composites; Figure S8: The magnetization of composites against the applied field at $300 \mathrm{~K}$ (a) MXCBCCu, (b) MXCS, (c) FC, (d) MXCBCCo, (e) MXCBCNi, (f) MXCBCFe, (g) MXCBCFe and (h) MXCBCFeO; Figure S9: Comparison of the TG and DTA of all the composites (a) MXCB, (b) MXCBCFeO, (c) MXCBCFe, (d) MXCBCNi, (e) MXCBCCo, (f) MXCS, (g) MXCBCCu, (h) FC, (i) MXCNTC25, (j) MXCNTNiC25, (k) MXCNTC10, (l) MXCNTC30 and (m) MC; Table S1: Elemental percentage of composites from EDX analysis; Table S2: Raman spectra band positions; Table S3: Atomic percentage MXene, MC, CNT, CNTO and composites from XPS analysis; Table S4: Comparison of EMI SE with thickness; Table S5: Comparison of maximum (MAX), minimum (MINI), average (AVE) shielding, SSE and SSE/t of the composite in each case; Table S6: Density of the composites; Table S7: Comparison of mass changes with different temperature range from TGA analysis; Table S8: Comparison of different peak positions range from DTA analysis.

Author Contributions: K.Y.C. and R.B. designed the project; K.R., B.M.K. and J.J.M. performed the experiments; L.R.L. and J.L. analyzed the data; S.B.L. supervised the analysis; K.R. wrote the manuscript.

Funding: This research was supported by the Leading Human Resource Training Program of Regional Neo industry through the National Research Foundation of Korea (NRF) funded by the Ministry of Science, ICT and future Planning (grant number) (NRF-2017H1D5A1043865).

Conflicts of Interest: The author declare no conflict of interest.

\section{References}

1. Pawar, S.P.; Rzeczkowski, P.; Potschke, P.; Krause, B.; Bose, S. Does the Processing Method Resulting in Different States of an Interconnected Network of Multiwalled Carbon Nanotubes in Polymeric Blend Nanocomposites Affect EMI Shielding Properties? ACS Omega 2018, 3, 5771-5782. [CrossRef]

2. Shahzad, F.; Alhabeb, M.; Hatter, C.B.; Anasori, B.; Hong, S.M.; Koo, C.M.; Gogotsi, Y. Electromagnetic interference shielding with 2D transition metal carbides (MXenes). Science 2016, 353, 1137-1140. [CrossRef] [PubMed] 
3. Cao, L.; Liu, G.; Li, J. Damage assessment of long-range rocket system by electromagnetic pulse weapon. AIP Conf. Proc. 2017, 1864, 020115.

4. Choi, G.; Shahzad, F.; Bahk, Y.M.; Jhon, Y.M.; Park, H.; Alhabeb, M.; Anasori, B.; Kim, D.S.; Koo, C.M.; Gogotsi, Y.; et al. Enhanced Terahertz Shielding of MXenes with Nano-Metamaterials. Adv. Opt. Mater. 2018, 6, 1701076. [CrossRef]

5. Pothupitiya Gamage, S.J.; Yang, K.; Braveenth, R.; Raagulan, K.; Kim, H.S.; Lee, Y.S.; Yang, C.M.; Moon, J.J.; Chai, K.Y. MWCNT coated free-standing carbon fiber fabric for enhanced performance in EMI shielding with a higher absolute EMI SE. Materials 2017, 10, 1350. [CrossRef]

6. Raagulan, K.; Braveenth, R.; Jang, H.; Seon Lee, Y.; Yang, C.M.; Mi Kim, B.; Moon, J.; Chai, K. Electromagnetic Shielding by MXene-Graphene-PVDF Composite with Hydrophobic, Lightweight and Flexible Graphene Coated Fabric. Materials 2017, 11, 1803. [CrossRef] [PubMed]

7. Lu, L.; Xing, D.; Teh, K.S.; Liu, H.; Xie, Y.; Liu, X.; Tang, Y. Structural effects in a composite nonwoven fabric on EMI shielding. Mater. Des. 2018, 120, 354-362. [CrossRef]

8. Al-Saleh, M.H.; Sundararaj, U. Electromagnetic interference shielding mechanisms of CNT/polymer composites. Carbon 2009, 47, 1738-1746. [CrossRef]

9. Yadav, R.S.; Kuřitka, I.; Vilcakova, J.; Skoda, D.; Urbánek, P.; Machovsky, M.; Masař, M.; Kalina, L.; Havlica, J. Lightweight $\mathrm{NiFe}_{2} \mathrm{O}_{4}$-Reduced Graphene Oxide-Elastomer Nanocomposite flexible sheet for electromagnetic interference shielding application. Compos. B Eng. 2019, 166, 95-111. [CrossRef]

10. Ravindren, R.; Mondal, S.; Nath, K.; Das, N.C. Synergistic effect of double percolated co-supportive MWCNT-CB conductive network for high-performance EMI shielding application. Polym. Adv. Technol. 2019, 1-12. [CrossRef]

11. Xu, Z.; Liang, M.; He, X.; Long, Q.; Yu, J.; Xie, K.; Liao, L. The preparation of carbonized silk cocoon-Co-graphene composite and its enhanced electromagnetic interference shielding performance. Compos. Part A Appl. Sci. Manuf. 2019, 119, 111-118. [CrossRef]

12. Wu, H.Y.; Jia, L.C.; Yan, D.X.; Gao, J.F.; Zhang, X.P.; Ren, P.G.; Li, Z.M. Simultaneously improved electromagnetic interference shielding and mechanical performance of segregated carbon nanotube/polypropylene composite via solid phase molding. Compos. Sci. Technol. 2018, 156, 87-94. [CrossRef]

13. Ma, X.; Shen, B.; Zhang, L.; Liu, Y.; Zhai, W.; Zheng, W. Porous superhydrophobic polymer/carbon composites for lightweight and self-cleaning EMI shielding application. Compos. Sci. Technol. 2018, 158, 86-93. [CrossRef]

14. Lu, S.; Shao, J.; Ma, K.; Chen, D.; Wang, X.; Zhang, L.; Meng, Q.; Ma, J. Flexible, mechanically resilient carbon nanotube composite films for high-efficiency electromagnetic interference shielding. Carbon 2018, 136, 387-394. [CrossRef]

15. Mondal, S.; Das, P.; Ganguly, S.; Ravindren, R.; Remanan, S.; Bhawal, P.; Das, T.K.; Das, N.C. Thermal-air ageing treatment on mechanical, electrical, and electromagnetic interference shielding properties of lightweight carbon nanotube based polymer nanocomposites. Compos. Pt A. Appl. Sci. Manuf. 2018, 107, 447-460. [CrossRef]

16. Lu, D.; Mo, Z.; Liang, B.; Yang, L.; He, Z.; Zhu, H.; Tang, Z.; Gui, X. Flexible, lightweight carbon nanotube sponges and composites for high-performance electromagnetic interference shielding. Carbon 2018, 133, 457-463. [CrossRef]

17. Liu, Y.F.; Feng, L.M.; Chen, Y.F.; Shi, Y.D.; Chen, X.D.; Wang, M. Segregated polypropylene/cross-linked poly (ethylene-co-1-octene)/multi-walled carbon nanotube nanocomposites with low percolation threshold and dominated negative temperature coefficient effect: Towards electromagnetic interference shielding and thermistors. Compos. Sci. Technol. 2018, 159, 152-161.

18. Alhabeb, M.; Maleski, K.; Anasori, B.; Lelyukh, P.; Clark, L.; Sin, S.; Gogotsi, Y. Guidelines for Synthesis and Processing of Two-Dimensional Titanium Carbide (Ti3C2Tx MXene). Chem. Mater. 2017, 29, 7633-7644. [CrossRef]

19. He, P.; Cao, M.; Shu, J.C.; Cai, Y.Z.; Wang, X.; Zhao, Q.; Yuan, J. Atomic Layer Tailoring Titanium Carbide MXene to Tune Transport and Polarization for Utilization of Electromagnetic Energy beyond Solar and Chemical Energy. ACS Appl. Mater. Interfaces 2019. [CrossRef]

20. Xiang, C.; Guo, R.; Lin, S.; Jiang, S.; Lan, J.; Wang, C.; Cui, C.; Xiao, H.; Zhang, Y. Lightweight and ultrathin $\mathrm{TiO}_{2}-\mathrm{Ti}_{3} \mathrm{C}_{2} \mathrm{~T}_{X} /$ graphene film with electromagnetic interference shielding. Chem. Eng. J. 2019, 360, 1158-1166. [CrossRef] 
21. Zhou, Z.; Liu, J.; Zhang, X.; Tian, D.; Zhan, Z.; Lu, C. Ultrathin MXene/Calcium Alginate Aerogel Film for High-Performance Electromagnetic Interference Shielding. Adv. Mater. Interfaces 2019, 1802040-1802049. [CrossRef]

22. Cui, C.; Xiang, C.; Geng, L.; Lai, X.; Guo, R.; Zhang, Y.; Xiao, H.; Lan, J.; Lin, S.; Jiang, S. Flexible and ultrathin electrospun regenerate cellulose nanofibers and $\mathrm{d}-\mathrm{Ti}_{3} \mathrm{C}_{2} \mathrm{~T}_{\mathrm{x}}$ (MXene) composite film for electromagnetic interference shielding. J. Alloys Compd. 2019, 785, 1246-1255. [CrossRef]

23. Li, X.; Yin, X.; Liang, S.; Li, M.; Cheng, L.; Zhang, L. 2D carbide MXene $\mathrm{Ti}_{2} \mathrm{CT}_{\mathrm{X}}$ as a novel high-performance electromagnetic interference shielding material. Carbon 2019, 146, 210-217. [CrossRef]

24. Raagulan, K.; Braveenth, R.; Jang, H.J.; Lee, Y.S.; Yang, C.M.; Kim, B.M.; Moon, J.J.; Chai, K.Y. Fabrication of Nonwetting Flexible Free-Standing MXene-Carbon Fabric for Electromagnetic Shielding in S-Band Region. Bull. Korean Chem. Soc. 2018, 39, 1412-1419. [CrossRef]

25. Georgakilas, V.; Gournis, D.; Tzitzios, V.; Pasquato, L.; Guldi, D.M.; Prato, M. Decorating carbon nanotubes with metal or semiconductor nanoparticles. J. Mater. Chem. 2017, 17, 2679-2694. [CrossRef]

26. Megiel, E. Surface modification using TEMPO and its derivatives. Adv. Colloid Interface Sci. 2017, 250, 158-184. [CrossRef] [PubMed]

27. Young, K.D. Bacterial morphology: Why have different shapes? Curr. Opin. Microbiol. 2017, 10, 596-600. [CrossRef] [PubMed]

28. Allen, J.W.; Sihanonth, P.; Gartz, J.; Toro, G. An ethnopharmacological and ethnomycological update on the occurrence, use, cultivation, chemical analysis, and SEM photography of neurotropic fungi from Thailand, Cambodia and other regions of South and Southeast Asia, Indonesia and Bali. Ethnomycological Journals: Sacred Mushroom Studies, 2012, 9, 1-129.

29. Clark, T.R.; Leonard, N.D.; Zhao, J.X.; Brodie, J.; McCook, L.J.; Wachenfeld, D.R.; Nguyen, A.D.; Markham, H.L.; Pandolfi, J.M. Historical photographs revisited: A case study for dating and characterizing recent loss of coral cover on the inshore Great Barrier Reef. Sci. Rep. 2016, 6, 19285. [CrossRef]

30. Yan, P.; Zhang, R.; Jia, J.; Wu, C.; Zhou, A.; Xu, J.; Zhang, X. Enhanced supercapacitive performance of delaminated two-dimensional titanium carbide/carbon nanotube composites in alkaline electrolyte. J. Power Sources 2015, 284, 38-43. [CrossRef]

31. Zhang, H.B.; Zhou, Y.C.; Bao, Y.W.; Li, M.S. Abnormal thermal shock behavior of $\mathrm{Ti}_{3} \mathrm{SiC}_{2}$ and $\mathrm{Ti}_{3} \mathrm{AlC}_{2}$. J. Mater. Res. 2006, 21, 2401-2407. [CrossRef]

32. Ivasyshyn, A.; Ostash, O.; Prikhna, T.; Podhurska, V.; Basyuk, T. Oxidation Resistance of Materials Based on Ti3AlC2 Nanolaminate at $600^{\circ} \mathrm{C}$ in Air. Nanoscale Res. Lett. 2016, 11, 358. [CrossRef] [PubMed]

33. Wang, L.; Zhang, H.; Wang, B.; Shen, C.; Zhang, C.; Hu, Q.; Zhou, A.; Liu, B. Synthesis and electrochemical performance of $\mathrm{Ti}_{3} \mathrm{C}_{2} \mathrm{~T}_{\mathrm{x}}$ with hydrothermal process. Electron. Mater. Lett. 2016, 12, 702-710. [CrossRef]

34. Han, M.; Yin, X.; Wu, H.; Hou, Z.; Song, C.; Li, X.; Zhang, L.; Cheng, L. Ti ${ }_{3} \mathrm{C}_{2}$ MXenes with modified surface for high-performance electromagnetic absorption and shielding in the X-band. ACS Appl. Mater. Interfaces 2016, 8, 21011-21019. [CrossRef]

35. Qian, A.; Hyeon, S.E.; Seo, J.Y.; Chung, C.H. Capacitance changes associated with cation-transport in free-standing flexible $\mathrm{Ti}_{3} \mathrm{C}_{2} \mathrm{~T}_{\mathbf{x}}(\mathrm{TO}, \mathrm{F}, \mathrm{OH}) \mathrm{MXene}$ film electrodes. Electrochim. Acta 2018, 266, 86-93. [CrossRef]

36. Saleh, T.A.; Agarwal, S.; Gupta, V.K. Synthesis of $\mathrm{MWCNT} / \mathrm{MnO}_{2}$ and their application for simultaneous oxidation of arsenite and sorption of arsenate. Appl. Catal. B Environ. 2011, 106, 46-53. [CrossRef]

37. Jia-Jia, C.; Xin, J.; Qiu-Jie, S.; Chong, W.; Qian, Z.; Ming-Sen, Z.; Quan-Feng, D. The preparation of nano-sulfur/MWCNTs and its electrochemical performance. Electrochim. Acta 2010, 55, 8062-8066. [CrossRef]

38. Gupta, V.K.; Agarwal, S.; Saleh, T.A. Synthesis and characterization of alumina-coated carbon nanotubes and their application for lead removal. J. Hazard. Mater. 2011, 185, 17-23. [CrossRef]

39. Ma, X.; Yu, J.; Wang, N. Glycerol plasticized-starch/multiwall carbon nanotube composites for electroactive polymers. Compos. Sci. Technol. 2008, 68, 268-273. [CrossRef]

40. Gupta, V.; Saleh, T.A. Syntheses of carbon nanotube-metal oxides composites; adsorption and photo-degradation. In Carbon Nanotubes-From Research to Applications; InTech: Shanghai, China, 2011.

41. użny, W.; Bańka, E. Relations between the structure and electric conductivity of polyaniline protonated with camphorsulfonic acid. Macromolecules 2000, 33, 425-429. [CrossRef]

42. Lorencová, L.; Bertok, T.; Dosekova, E.; Holazová, A.; Paprckova, D.; Vikartovská, A.; Sasinková, V.; Filip, J.; Kasák, P.; Jerigová, M.; et al. Electrochemical performance of Ti3C2Tx MXene in aqueous media: Towards ultrasensitive $\mathrm{H}_{2} \mathrm{O}_{2}$ sensing. Electrochim. Acta 2017, 235, 471-479. [CrossRef] [PubMed] 
43. Wang, X.; Garnero, C.; Rochard, G.; Magne, D.; Morisset, S.; Hurand, S.; Chartier, P.; Rousseau, J.; Cabioc'h, T.; Coutanceau, $\mathrm{C}$; et al. A new etching environment $\left(\mathrm{FeF}_{3} / \mathrm{HCl}\right)$ for the synthesis of two-dimensional titanium carbide MXenes: A route towards selective reactivity vs. water. J. Mater. Chem. A 2017, 5, 22012-22023. [CrossRef]

44. Yan, J.; Ren, C.E.; Maleski, K.; Hatter, C.B.; Anasori, B.; Urbankowski, P.; Sarycheva, A.; Gogotsi, Y. Flexible MXene/graphene films for ultrafast supercapacitors with outstanding volumetric capacitance. Adv. Funct. Mater. 2017, 27, 1701264. [CrossRef]

45. Sui, X.M.; Giordani, S.; Prato, M.; Wagner, H.D. Effect of carbon nanotube surface modification on dispersion and structural properties of electrospun fibers. Appl. Phys. Lett. 2009, 95, 233113. [CrossRef]

46. Mishra, A.K.; Ramaprabhu, S. Magnetite decorated multiwalled carbon nanotube based supercapacitor for arsenic removal and desalination of seawater. J. Phys. Chem. C 2017, 114, 2583-2590. [CrossRef]

47. He, Y.; Huang, L.; Cai, J.S.; Zheng, X.M.; Sun, S.G. 2010. Structure and electrochemical performance of nanostructured $\mathrm{Fe}_{3} \mathrm{O}_{4}$ /carbon nanotube composites as anodes for lithium ion batteries. Electrochimica Acta 2010, 55, 1140-1144. [CrossRef]

48. Grassi, G.; Scala, A.; Piperno, A.; Iannazzo, D.; Lanza, M.; Milone, C.; Pistone, A.; Galvagno, S. A facile and ecofriendly functionalization of multiwalled carbon nanotubes by an old mesoionic compound. Chem. Comm. 2012, 48, 6836-6838. [CrossRef]

49. Mali, S.S.; Betty, C.A.; Bhosale, P.N.; Patil, P.S. Synthesis, characterization of hydrothermally grown MWCNT- $\mathrm{TiO}_{2}$ photoelectrodes and their visible light absorption properties. ECS J. Solid State Sci. Technol. 2012, 1, M15-M23. [CrossRef]

50. Dong, C.K.; Li, X.; Zhang, Y.; Qi, J.Y.; Yuan, Y.F. $\mathrm{Fe}_{3} \mathrm{O}_{4}$ nanoparticles decorated multi-walled carbon nanotubes and their sorption properties. Chem. Res. Chin. Univ. 2009, 25, 936-940.

51. Jiao, Z.; Qiu, J. Microwave absorption performance of iron oxide/multiwalled carbon nanotubes nanohybrids prepared by electrostatic attraction. J. Mater. Sci. 2018, 53, 3640-3646. [CrossRef]

52. Crane, R.A.; Scott, T.B. The Effect of Vacuum Annealing of Magnetite and Zero-Valent Iron Nanoparticles on the Removal of Aqueous Uranium. J. Nanotechnol. 2013, 2013, 11. [CrossRef]

53. Jiang, Z.; Xie, J.; Jiang, D.; Wei, X.; Chen, M. Modifiers-assisted formation of nickel nanoparticles and their catalytic application to p-nitrophenol reduction. CrystEngComm 2013, 15, 560-569. [CrossRef]

54. Balela, M.D.L.; Yagi, S.; Lockman, Z.; Aziz, A.; Amorsolo, A.V.; Matsubara, E. 2009. Electroless deposition of ferromagnetic cobalt nanoparticles in propylene glycol. J. Electrochem. Soc. 2009, 156, E139-E142. [CrossRef]

55. Betancourt-Galindo, R.; Reyes-Rodriguez, P.Y.; Puente-Urbina, B.A.; Avila-Orta, C.A.; Rodríguez-Fernández, O.S.; Cadenas-Pliego, G.; Lira-Saldivar, R.H.; García-Cerda, L.A. Synthesis of copper nanoparticles by thermal decomposition and their antimicrobial properties. J. Nanomater. 2014, 2014, 10. [CrossRef]

56. Graat, P.; Somers, M.A. Quantitative analysis of overlapping XPS peaks by spectrum reconstruction: Determination of the thickness and composition of thin iron oxide films. Surface and Interface Analysis: An International Journal devoted to the development and application of techniques for the analysis of surfaces. Interfaces Thin Films 1998, 26, 773-782.

57. Lee, T.W.; Lee, S.E.; Jeong, Y.G. Carbon nanotube/cellulose papers with high performance in electric heating and electromagnetic interference shielding. Compos. Sci. Technol. 2016, 131, 77-87. [CrossRef]

58. Datsyuk, V.; Kalyva, M.; Papagelis, K.; Parthenios, J.; Tasis, D.; Siokou, A.; Kallitsis, I.; Galiotis, C. Chemical oxidation of multiwalled carbon nanotubes. Carbon 2008, 46, 833-840. [CrossRef]

59. Halim, J. Synthesis and Characterization of 2D Nanocrystals and Thin Films of Transition Metal Carbides (MXenes). Doctoral Dissertation, Linköping University Electronic Press, Linköping, Sweden, 2014.

60. Shen, C.; Wang, L.; Zhou, A.; Wang, B.; Wang, X.; Lian, W.; Hu, Q.; Qin, G.; Liu, X. Synthesis and Electrochemical Properties of Two-Dimensional RGO/ $\mathrm{Ti}_{3} \mathrm{C}_{2} \mathrm{~T}_{\mathrm{x}}$ Nanocomposites. Nanomaterials 2018, 8, 80. [CrossRef]

61. Shah, S.A.; Habib, T.; Gao, H.; Gao, P.; Sun, W.; Green, M.J.; Radovic, M. Template-free 3D titanium carbide $\left(\mathrm{Ti}_{3} \mathrm{C}_{2} \mathrm{~T}_{\mathrm{x}}\right) \mathrm{MXene}$ particles crumpled by capillary forces. Chem. Commun. 2017, 53, 400-403. [CrossRef] [PubMed]

62. Liu, J.; Zhang, H.B.; Sun, R.; Liu, Y.; Liu, Z.; Zhou, A.; Yu, Z.Z. Hydrophobic, Flexible, and Lightweight MXene Foams for High-Performance Electromagnetic-Interference Shielding. Adv. Mater. 2017, 29, 1702367. [CrossRef] [PubMed] 
63. Ibrahim, S.F.; El-Amoudy, E.S.; Shady, K.E. Thermal analysis and characterization of some cellulosic fabrics dyed by a new natural dye and mordanted with different mordants. Int. J. Chem. 2011, 3, 40. [CrossRef]

64. Taira, M.; Araki, Y. DTG thermal analyses and viscosity measurements of three commercial agar impression materials. J. Oral Rehabil. 2002, 29, 697-701. [CrossRef] [PubMed]

65. Kushwaha, A.; Aslam, M. Roughness enhanced surface defects and photoconductivity of acid etched ZnO nanowires. In Proceedings of the 2012 International Conference on Emerging Electronics, Mumbai, India, 15-17 December 2012; pp. 1-4.

66. Zhao, M.Q.; Ren, C.E.; Ling, Z.; Lukatskaya, M.R.; Zhang, C.; Van Aken, K.L.; Barsoum, M.W.; Gogotsi, Y. Flexible MXene/carbon nanotube composite paper with high volumetric capacitance. Adv. Mater. 2015, 27, 339-345. [CrossRef] [PubMed]

67. Kim, B.C.; Innis, P.C.; Wallace, G.G.; Low, C.T.J.; Walsh, F.C.; Cho, W.J.; Yu, K.H. Electrically conductive coatings of nickel and polypyrrole/poly (2-methoxyaniline-5-sulfonic acid) on nylon Lycra®textiles. Prog. Org. Coat. 2013, 76, 1296-1301. [CrossRef]

68. Zhang, L.; Zhang, Y. Fabrication and magnetic properties of $\mathrm{Fe}_{3} \mathrm{O}_{4}$ nanowire arrays in different diameters. Journal of Magnetism and Magnetic. Materials 2009, 321, L15-L20.

69. Iida, H.; Takayanagi, K.; Nakanishi, T.; Osaka, T. Synthesis of $\mathrm{Fe}_{3} \mathrm{O}_{4}$ nanoparticles with various sizes and magnetic properties by controlled hydrolysis. J. Colloid Interface Sci. 2007, 314, 274-280. [CrossRef] [PubMed]

70. Chang, H.; Su, H.T. Synthesis and magnetic properties of Ni nanoparticles. Rev. Adv. Mater. Sci. 2008, 18, 667-675.

71. Alborzi, Z.; Hassanzadeh, A.; Golzan, M.M. Superparamagnetic behavior of the magnetic hysteresis loop in the Fe2O3@ Pt core-shell nanoparticles. Int. J. Nanosci. Nanotechnol. 2012, 8, 93-98.

72. Hu, C.; Gao, Z.; Yang, X. Fabrication and magnetic properties of Fe3O4 octahedra. Chem. Phys. Lett. 2006, 429, 513-517. [CrossRef]

73. Xing, D.; Lu, L.; Teh, K.S.; Wan, Z.; Xie, Y.; Tang, Y. Highly flexible and ultra-thin Ni-plated carbon-fabric/ polycarbonate film for enhanced electromagnetic interference shielding. Carbon 2018, 132, 32-41. [CrossRef]

74. Xu, Y.; Yang, Y.; Duan, H.; Gao, J.; Yan, D.X.; Zhao, G.; Liu, Y. Flexible and highly conductive sandwich nylon/nickel film for ultra-efficient electromagnetic interference shielding. Appl. Surf. Sci. 2018, 455, 856-863. [CrossRef]

75. Bagotia, N.; Mohite, H.; Tanaliya, N.; Sharma, D.K. A comparative study of electrical, EMI shielding and thermal properties of graphene and multiwalled carbon nanotube filled polystyrene nanocomposites. Polym. Compos. 2018, 39, E1041-E1051. [CrossRef]

76. Cao, W.T.; Chen, F.F.; Zhu, Y.J.; Zhang, Y.G.; Jiang, Y.Y.; Ma, M.G.; Chen, F. Binary Strengthening and Toughening of MXene/Cellulose Nanofiber Composite Paper with Nacre-Inspired Structure and Superior Electromagnetic Interference Shielding Properties. ACS Nano 2018, 12, 4583-4593. [CrossRef] [PubMed]

77. Lodhi, G.; Kim, Y.S.; Hwang, J.W.; Kim, S.K.; Jeon, Y.J.; Je, J.Y.; Ahn, C.B.; Moon, S.H.; Jeon, B.T.; Park, P.J. Chitooligosaccharide and its derivatives: Preparation and biological applications. BioMed Res. Int. 2014, 2014, 654913. [CrossRef] [PubMed]

(C) 2019 by the authors. Licensee MDPI, Basel, Switzerland. This article is an open access article distributed under the terms and conditions of the Creative Commons Attribution (CC BY) license (http:/ / creativecommons.org/licenses/by/4.0/). 